\title{
Power Allocation for D2D Communications Using Max-Min Message-Passing Algorithm
}

\author{
Salma Kazemi Rashed, Reza Asvadi, Senior Member, IEEE, Siavash Rajabi, Seyed Ali Ghorashi, Senior \\ Member, IEEE and Maria G. Martini, Senior Member, IEEE
}

\begin{abstract}
The approach of factor-graphs (FGs) is applied in the context of power control and user pairing in Device-toDevice (D2D) communications as an effective underlay concept in wireless cellular networks. D2D communications can increase the spectral efficiency of wireless cellular networks by establishing a direct link between devices with limited help from the evolved node base stations (eNBs). A well-designed user pairing and power allocation scheme with low complexity can remarkably improve the system's performance. In this paper, a simple and distributed FG based approach is utilized for power control and user pairing implementation in an underlay cellular network with D2D communications. A max-min criterion is proposed to maximize the minimum rate of all active users in the network, including the cellular and multiple D2D co-channel links in the uplink direction. An associated message-passing (MP) algorithm is presented to distributedly solve the resultant NP-hard maximization problem, with a guaranteed convergence compared to game theoretic and Q-learning based methods. The complexity and convergence of the proposed method is analyzed and numerical results confirm that the proposed scheme outperforms alternative algorithms in terms of complexity, while keeping the sum-rate of users nearly the same as centralized counterpart methods.
\end{abstract}

Index Terms-Device-to-device (D2D) communications, factorgraph (FG), Max-Min, message-passing.

\section{INTRODUCTION}

To meet the need for high data rate communications, new technologies have been developed in the last decade. Among them, device-to-device (D2D) communications is a short range and low-power mechanism that enables two nearby devices to connect with each other without the help of evolved node base station (eNB). It plays an important role in the realization of high data rate local services. D2D systems can be

Copyright (c) 2015 IEEE. Personal use of this material is permitted. However, permission to use this material for any other purposes must be obtained from the IEEE by sending a request to pubs-permissions@ieee.org.

S. K. Rashed and R. Asvadi are with Cognitive Telecommunication Research Group, Faculty of Electrical Engineering, Shahid Beheshti University, 1983963113, Tehran, Iran. (e-mails: salma.kazemi_rashed@med.lu.se; r_asvadi@sbu.ac.ir).

S. Rajabi is with Department of Electrical Engineering, Hamedan University of Technology, Hamedan 65155, Iran. (e-mail: siavash.rajabi@hut.ac.ir)

S. A. Ghorashi is with School of Architecture, Computing and Engineering, University of East London, London, UK. He is also with Cognitive Telecommunication Research Group, Faculty of Electrical Engineering, Shahid Beheshti University, 1983963113, Tehran, Iran. (email: a_ghorashi@sbu.ac.ir (Corresponding Author))

M. G. Martini is with Wireless Multimedia Networking Research Group, Kingston University, London KT1 2EE, U.K. (e-mail: m.martini@kingston.ac.uk).

This work was partially supported by EPSRC Grant EP/P022715/1 - The Internet of Silicon Retinas (IOSIRE): Machine to machine communications for neuromorphic vision sensing data. categorized into in-band (underlay and overlay) and out-band (autonomous and controlled) [1]-[4]. Most of the research is focused on the underlay in-band D2D communications, which means that D2D devices and a cellular network use the same frequency band. These systems do not need a new infrastructure, separated security and control considerations from the cellular network and have a high network resource efficiency [1]. Therefore, in this paper we focus on a cellular network empowered by underlay and in-band D2D communications with the main challenge of interference of D2D users on cellular users as well as the interference on other D2D pairs. There are two modes of resource allocation mechanisms specified in the 3GPP standard [5]: (a) distributed resource allocation from an allocated resource pool(s), and (b) eNB related resource allocation mechanisms. Some of the solutions, proposed in the literature to mitigate the interference issue, impose a huge signaling overhead over the cellular network [6]-[8]. Therefore, the recent research goes toward distributed resource allocation of $\mathrm{D} 2 \mathrm{D}$ communications to address the problem whenever an associated Base Station (BS) is assumed to have either a part of or no information about the network [9], [10]. In these cases, the power allocation or joint resource allocation and power control solutions are carried out at the device level, like the studies recently performed in [9], [11][19]. Alternatively, these allocations can be made in a hybrid form, partly performed in the eNB in a centralized manner and partly at device level [20]. Recent studies regarding D2D communications are summarized in TABLE I. In some schemes of this table, the entire network information has to be available at the BS. This is impractical in dense networks because in such cases the BSs should carry a huge signaling and computational overhead while D2D communications occur. In this paper, we focus on the distributed resource allocation schemes which are at device level. Device discovery and power allocation in two modes of "partial-in-network" and "out-of-network" coverage are also recommended in LTEA standard [21], [22]. To apply this recommendation, some distributed solutions have been established by using gametheoretic and Q-learning based methods [9], [11]-[19], [23]. These studies usually benefit from the local information of other users by utilizing an estimation or a cooperation among them. Their assumptions lead to an imperfect knowledge of network in realistic scenarios, which imposes a limitation on the convergence to Nash equilibrium, a reduction in precision of optimal solutions, and a delay in converging to equilibrium points. Moreover, the mentioned approaches also maximize the sum-rate of a cellular network and do not always result in 
TABLE I

EXISTING SCHEMES.

\begin{tabular}{|c|c|c|c|c|c|}
\hline Ref. & Achievement & Model & Algorithm & Mechanism & Drawback \\
\hline [7] & $\begin{array}{l}\text { Power-channel allocation in D2D } \\
\text { network }\end{array}$ & Markov Decision process & Explorative Q-learning & Centralized & Huge overhead \\
\hline [9], [18] & Joint channel-power allocation & Stackelberg game with pricing & Stochastic learning algorithm & Distributed & $\begin{array}{l}\text { Limitations to achieve Nash-equilibrium and the local } \\
\text { optimality of achieved NE }\end{array}$ \\
\hline [11] & $\begin{array}{l}\text { Joint power control and mode } \\
\text { selection }\end{array}$ & $\begin{array}{l}\text { A system throughput } \\
\text { maximization problem subject to } \\
\text { the QoS of users }\end{array}$ & A distributed partly iterative algorithm & Distributed & $\begin{array}{l}\text { The potentially high interference caused to the surrounding } \\
\text { links is ignored. }\end{array}$ \\
\hline [12] & Power allocation & - & A non-cooperative power control game & Distributed with the BS control & $\begin{array}{c}\text { Sharing channel information and prices among users proposed } \\
\text { a large signaling overhead }\end{array}$ \\
\hline [13] & Power and mode selection & A Bayesian Network (BN) & A probabilistic approach & $\begin{array}{l}\text { Distributed using local } \\
\text { information }\end{array}$ & $\begin{array}{c}\text { Not seeking a global optimal solution for the limitation of } \\
\text { local information }\end{array}$ \\
\hline [14] & $\begin{array}{l}\text { Resource allocation and power } \\
\text { control }\end{array}$ & - & $\begin{array}{l}\text { A Q-learning based algorithm with a } \\
\text { exploitative cache content management policy }\end{array}$ & Local distributed & Different application \\
\hline [15] & Channel allocation & - & $\begin{array}{l}\text { a Distributed receiver-oriented channel } \\
\text { allocation algorithm followed by a sender-jump } \\
\text { blind channel rendezvous algorithm }\end{array}$ & Distributed & Different application \\
\hline [16] & Power allocation & A potential game & Fully distributed iterative algorithm & Distributed & $\begin{array}{l}\text { Limitation of achieved NE and not applicable for dense } \\
\text { network for using broadcast sounding signal instead of } \\
\text { message passing or channel gain estimations. }\end{array}$ \\
\hline [17] & Power control & $\begin{array}{l}\text { A mean-field game theoretic } \\
\text { framework with the } \\
\text { two-dimensional dynamics }\end{array}$ & $\begin{array}{l}\text { A novel energy and interference aware power } \\
\text { control policy based on the Lax-Friedrichs } \\
\text { scheme and the Lagrange relaxation }\end{array}$ & Distributed & $\begin{array}{l}\text { There is a need to store historical measured information of } \\
\text { interference of dynamics as well as energy availability }\end{array}$ \\
\hline [19] & Channel and power allocation & $\begin{array}{l}\text { A non-convex optimization } \\
\text { problem and a Stackelberg game } \\
\text { with pricing }\end{array}$ & $\begin{array}{l}\text { A centralized convex solution and a } \\
\text { decentralized BS-coordinated solution }\end{array}$ & $\begin{array}{l}\text { Centralized and a BS-supervised } \\
\text { distributed scheme }\end{array}$ & $\begin{array}{l}\text { Unrealistic information availability in BS assumptions and } \\
\text { challenge of determining optimal pricing amount }\end{array}$ \\
\hline [20] & $\begin{array}{l}\text { Hybrid centralized-distributed } \\
\text { resource (power-channel) } \\
\text { allocation for D2D underlay } \\
\text { networks } \\
\end{array}$ & $\begin{array}{l}\text { Partly a weighted matching } \\
\text { problem and partly a potential } \\
\text { game }\end{array}$ & $\begin{array}{l}\text { Hungarian algorithm for matching part and } \\
\text { Q-learning for the power control game }\end{array}$ & Hybrid (centralized-distributed) & Overhead in terms of signaling and computation at the BS \\
\hline [23] & $\begin{array}{l}\text { Bandwidth allocation to D2D } \\
\text { users }\end{array}$ & A non-cooperative game & $\begin{array}{l}\text { A repeated bandwidth allocation algorithm with } \\
\text { incomplete channel state information }\end{array}$ & Centralized & Ignorance of inter-cell interference and subchannel allocation \\
\hline
\end{tabular}

a fair rate allocation to all devices.

Among distributed schemes, message-passing (MP) algorithms are recognized as powerful tools that can efficiently adapt to many problems in communications theory such as coding, e.g., Sparse Code Multiple Access (SCMA), resource allocation, localization [24]-[30] and recently, channel estimation of massive MIMO networks to infer the posterior probabilities of model parameter statistics in the context of optimal Bayesian Kalman filter and Bayesian Learning [31], [32]. In most of the mentioned works, the MP algorithm is used to overcome the computational challenges of the highdimensional integrals in computing the marginal distributions in order to calculate the posterior joint PDF of unknown parameters. Thus, the factor-graph (FG) based sum-product algorithm, known as Belief Propagation, is used to infer the posteriors of the target parameters.

In contrast, applying MP algorithms in power allocation problems provides a reduced-complexity solution for an NPhard optimization problem without the need for a central decision maker. Actually, calculation of the marignal probabilities is not the aim of utilizing MP algorithms in a power allocation problem. Specifically, by utilizing an FG model for MP algorithms, the network-wide optimization problems are decomposed into multiple optimization problems performed at each device. Furthermore, the passing messages among devices satisfies the optimization constraints via introducing additional checks in the factor nodes. The MP methods were applied for the D2D resource allocation, under different circumstances like multi-carrier multi-format systems [28], relayaided cellular networks [29], and joint user-association and resource allocation [30].

In [28] two novel distributed resource allocation schemes are proposed based on MP methods for the uplink of a cellular system. Cooperation between users and eNB leads to a better network performance than that of the centralized schemes. Also, a low complexity distributed resource allocation is proposed in [29] for relay-assisted D2D communications via maximizing the network sum-rate by converting the problem to a max-sum MP problem over a graphical model.

With the aim of maximizing the network sum-rate, a novel belief propagation algorithm is proposed in [30] to jointly optimize user association, sub-channel assignment, and power allocation to address the inter-cell interference coordination problem in a heterogeneous cellular network. In general, there is no guarantee for the convergence of the MP algorithms in FGs with cycles (e.g., [28]), and even those with the converged solutions do not guarantee to achieve the global optimality of the joint user association and sub-channel selection problem (e.g., [30]).

To address the shortcomings of centralized and other distributed schemes, we devise a distributed joint power allocation and pairing selection scheme by employing a max-min MP algorithm. Hence, by limiting the role of eNB in power control and pairing of D2D pairs, the NP-hard problem of pairing the D2D users is broken to a novel distributed method with a tractable complexity. Additionally, the max-min MP algorithm preserves the advantages of centralized methods as well as the fairness in power allocation. In principle, there is a compromise between the maximization of sum-rate and the fairness. It means that the system's fairness will be degraded by optimizing the sum-rate. In contrast, it will reach the maximum value if the sum-rate diminishes. In general, a fair resource allocation can be considered with optimizing the sum-rate by allocation of adequate resources to those users with better channel conditions or on the other hand, such an allocation that does not cause to starve users with weak channel conditions. In this paper, we propose a solution to maximize the minimum rate of all active D2D pairs and the cellular links ${ }^{1}$. Applying the proposed MP algorithm leads to a relatively fair allocation of resources among the users which is confirmed by some numerical results. By approximating the

\footnotetext{
${ }^{1}$ Although we follow the LTE-A standard in this paper, the proposed approach can be applied to $5 \mathrm{G}$ networks with next generation NodeBs (gNBs) in general.
} 


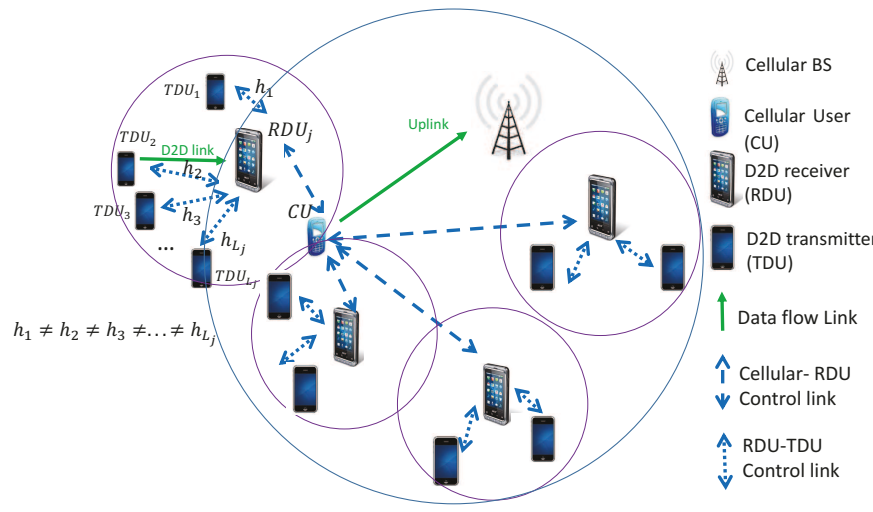

Fig. 1. System model.

constraints of the main problem and the log transformation of variables [33], we convert the problem into a convex optimization one. Thus, a distributed low complexity solution is proposed to solve the problem. Furthermore, we analyze the convergence and complexity of the proposed method in the presumed system model.

The organization of this paper is as follows. In Section II, system model and problem formulation are described in details. In Section III, an FG based distributed MP solution for power control and the best pair selection of D2D are presented. Variable nodes, factor nodes and structured messages are described in this part as well. Complexity and convergence analysis of the proposed method are also discussed in this section. Section IV is dedicated to the simulation results, and finally Section $\mathrm{V}$ concludes the paper.

Throughout the paper, max-min and minimum rate maximization, as well as max-sum and sum-rate maximization, are used interchangeably. $D, B$, and $C$ are the abbreviations standing for the D2D, Base-station, and Cellular, respectively, and $R$ and $T$ are the abbreviations for Receiver and Transmitter in the superscripts and subscripts of the variables. Some variables and functions have one of these abbreviations to discriminate their types. Scalar random variables and vectors are illustrated by upper-case italic letters, e.g., $X$ and bold-faced italic letters, e.g., $\boldsymbol{x}$, respectively. Furthermore, the realization of random variables and function definitions are indicated by lower-case italic letters, e.g., $x$. The $i$-th element of vector $\boldsymbol{x}$ is described as $x_{i}$. In almost all equations, a small letter subscript is used as the distinguishing index, e.g., $X_{k}$ and $x_{k}($.$) . Arrows in the$ subscript of some variables present the direction of exchanged messages $\boldsymbol{x}_{i \rightarrow j}$ and $|X|$ stands for the number of possible values of variable $X$. Furthermore, $\max _{x_{1}, \ldots, x_{N} \sim x_{j}} f\left(x_{1}, \ldots, x_{N}\right)$ denotes maximization of a function over all $x_{j}$ 's, $1 \leq j \leq N$, except the variable(s) after $\sim$, i.e., $x_{j}$. Also, if the argument variables are known from the context, they are omitted.

\section{PRELIMINARIES}

\section{A. System Model}

We consider a D2D communication network, working underlay of a cellular wireless network where multiple cellular users can be active in multiple orthogonal channels with the multiple interferer D2D pairs in the same channel. However, in order to avoid the complexity of a big network, we only investigate a single cell of a wireless network including a cellular link as well as multiple D2D co-channel links in uplink direction. However, each of these cellular users forms its own cycle-free FG and works in a parallel form along with other cellular users. Therefore, we have a forest ${ }^{2}$ of FGs in which they work independently from each other. Thus, we have only selected and solved one of these allocation problems that might be repeated for all other cellular users and other cells as well. This could be verified with orthogonality of cellular users in adjacent cells or imposing a maximum transmitting power in each cellular channel of the adjacent cells. Each D2D link comprises a receiver and multiple potential transmitters that contain the desired content of that receiver. The proposed model is depicted in Fig. 1 in which transmitters are assumed to be uniformly distributed in a circle with the center of the aforementioned receiver. We also suppose that the transmitters of each D2D link and their contents are different from each other. Therefore, the devices across different D2D links do not need to communicate. A distance-based power allocation is considered in this model which means that a minimum predefined distance is presumed between the receivers of all co-channel D2D links and the cellular one. Therefore, the assumption of no communication between co-channel D2D links is reasonable. In this paper, our goal is to find the best transmitter for each receiver in every D2D link, which we call "pairing". In addition, the optimal power levels of active transmitters are calculated by applying a fairness criterion. The list of mathematical notations used in this paper is given in Table II.

\section{B. Problem Formulation}

The proposed optimization problem, with the given Quality of Service (QoS) and the power constraints of D2D and cellular links, is formulated in (1). The SINR of D2D links and the cellular link are indicated by $\gamma_{j k}^{D}$ and $\gamma^{C}$, respectively. The minimum data rate of all active cellular and D2D links in the network is defined by $R_{0}$ which depends on the power levels of cellular and D2D transmitters. The rate of each active link is constrained to be greater than $R_{0}$ through (1b) and (1c). Moreover, the QoS of active links are considered through (1d) and (1e) inequalities. Additionally, the limitations of scheduling options for power levels are determined through

\footnotetext{
${ }^{2}$ Cycle-free connected graph is called "tree" and multiple "trees" is called "forest" in the graph theory.
} 
TABLE II

NOTATION SUMMARY

\begin{tabular}{|c|c|}
\hline$N$ & Number of all potential D2D receivers that are co-channel with the specific cellular user \\
\hline$j$ & Index of a potential D2D receiver \\
\hline$L_{j}$ & Number of all potential D2D transmitters around the specific D2D receiver $j$ \\
\hline$k_{j}$ & Index of a potential D2D transmitter $k$ around the receiver $j$ \\
\hline $\mathcal{D}^{R}=\{j \mid j=1,2, \ldots, N\}$ & Set of all potential D2D receivers that are co-channel with the specific cellular user \\
\hline $\mathcal{D}_{j}^{T}=\left\{k_{j} \mid k_{j}=1,2, \ldots, L_{j}\right\}$ & Set of all potential D2D transmitters around the specific D2D receiver $j$ \\
\hline$\gamma^{C}$ & SINR of cellular link \\
\hline$\gamma_{j k}^{D}$ & SINR of a D2D link between D2D transmitter $k_{j}$ to the D2D receiver $j$ \\
\hline$R_{0}$ & Minimum data rate of all active cellular and D2D links in the network \\
\hline$P^{C}, P_{j k}^{D}$ & Power of cellular link (uplink) and D2D transmitter $k_{j}$ \\
\hline$h_{B, C}, d_{B, C}$ & Small scale fading coef. and distance between eNB and cellular user \\
\hline$h_{j k, j}, d_{j k, j}$ & Small scale fading coef. and distance between D2D transmitter $k_{j}$ and D2D receiver $j$ \\
\hline$\alpha$ & Path loss exponent \\
\hline$\rho$ & Binary power allocation vector \\
\hline$\rho_{j}^{\prime}$ & Binary pairing vector at $\mathrm{D} 2 \mathrm{D}$ receiver $j$ \\
\hline$\gamma_{\min }^{C}, \gamma_{\min }^{D}$ & Minimum acceptable SINR of cellular and D2D links \\
\hline$P_{\max }^{C}, P_{\max }^{D}$ & Maximum power constraints of cellular and D2D users \\
\hline$N_{0}$ & Noise power \\
\hline$\ell_{\max }$ & Maximum number of iterations \\
\hline $\boldsymbol{\mu}_{g_{j k} \rightarrow P_{j k}^{D}}^{(\ell)}$ & Output messages of FG's Layer4 toward Layer3 (Fig. 2) at $\ell$-th iteration sent by D2D TDUs \\
\hline $\boldsymbol{\mu}_{f_{j} \rightarrow P^{C}}^{(\ell)}$ & Output messages of FG's Layer2 toward Layer1 (Fig. 2) at $\ell$-th iteration sent by D2D RDUs \\
\hline $\boldsymbol{\mu}_{f_{j} \rightarrow P_{j k}^{D}}^{(\ell)}$ & Output messages of FG's Layer2 toward Layer3 (Fig. 2) at $\ell$-th iteration sent by D2D RDUs \\
\hline
\end{tabular}

(1f) and (1g).

$$
\begin{array}{cl}
\underset{P^{C}, P_{j k}^{D}}{\operatorname{maximize}} & R_{0} \\
\text { subject to } & \log _{2}\left(1+\gamma^{C}\right) \geq R_{0} \\
& \log _{2}\left(1+\gamma_{j k}^{D}\right) \geq R_{0}, \quad \forall j \in \mathcal{D}^{R}, \forall k \in \mathcal{D}^{T} \\
& R_{0} \geq \log _{2}\left(1+\gamma_{\min }^{C}\right) \\
& R_{0} \geq \log _{2}\left(1+\gamma_{\min }^{D}\right) \\
& 0 \leq P^{C} \leq P_{\max }^{C}, \\
& 0 \leq P_{j k}^{D} \leq P_{\max }^{D}, \quad \forall j \in \mathcal{D}^{R}, \forall k \in \mathcal{D}^{T}
\end{array}
$$

where $\gamma^{C}$ and $\gamma_{j k}^{D}$ are formulated in (2) and (3), respectively [29].

$$
\begin{aligned}
& \gamma^{C}\left(P^{C}, P_{j k}^{D}\right)=\frac{P^{C} h_{B, C}^{2} d_{B, C}^{-\alpha}}{\sum_{j=1}^{N} \rho_{j} \sum_{k=1}^{L_{j}} \rho^{\prime}{ }_{j k} P_{j k}^{D} h_{j k, C}^{2} d_{j k, C}^{-\alpha}+N_{0}} \\
& \gamma_{j k}^{D}\left(P_{j k}^{D}, P^{C}\right)= \\
& \frac{P_{j k}^{D} h_{j k, j}^{2} d_{j k, j}^{-\alpha}}{P^{C} h_{B, j}^{2} d_{B, j}^{-\alpha}+\sum_{\substack{j^{\prime}=1 \\
j^{\prime} \neq j}}^{N} \rho_{j^{\prime}} \sum_{\substack{k_{j}^{\prime}=1 \\
k^{\prime} \neq k}}^{L_{j}} \rho^{\prime}{ }_{j^{\prime} k^{\prime}} P_{j^{\prime} k^{\prime}}^{D} h_{j^{\prime} k^{\prime}, j}^{2} d_{j^{\prime} k^{\prime}, j}+N_{0}} \text {, }
\end{aligned}
$$

where $\gamma_{j k}^{D}$ and $\gamma^{C}$ depend on the power of incoming desired signal, interference power of interfering faded signals, and some noise term. The active D2D links and pairing status of D2D transmitters and receivers are indicated by the binary vectors $\boldsymbol{\rho}$ and $\boldsymbol{\rho}_{j}^{\prime}$. There are two main groups of variables in the max-min problem (1); one of them is pointed out clearly in (1f) and (1g) as cellular and D2D transmitter powers, respectively. The other group of variables is composed of binary power allocation vector -denoted by $\boldsymbol{\rho}$ - and also binary pairing vector -denoted by $\boldsymbol{\rho}_{j}^{\prime}$ - at D2D receiver $j$ which are implicitly mentioned in (1). The presence of these binary vectors as objective parameters, set the problem in the category of NPhard problems. However, if the binary power allocation and pairing vectors ( $\boldsymbol{\rho}$ and $\boldsymbol{\rho}_{j}^{\prime}$, respectively) are assigned with predefined values, then (1) turns into a non-binary optimization problem. Thus, in this paper a pre-evaluation is performed on the binary vectors and their effect is implicitly expressed in the power variables as $P_{j k}^{D}=0$ if $\rho_{j k}^{\prime}=0$ and for all $k_{j} \in \mathcal{D}_{j}^{T}, P_{j k}^{D}=0$ if $\rho_{j}=0$.

The other challenge of (1) would be the challenge of nonconvex constraints (1b) and (1c). However, if the two most likely conditions of $\gamma^{C} \gg 1$ and $\gamma_{j k}^{D} \gg 1$ are considered, the non-convex constraints can be changed to (4a) and (4b), respectively:

$$
\begin{aligned}
& \log _{2}\left(\gamma^{C}\right) \geq R_{0} \\
& \log _{2}\left(\gamma_{j k}^{D}\right) \geq R_{0} .
\end{aligned}
$$

The reason is that the inequalities (1b) and (1c) are changed to the convex functions with an exponential power transformation by using (4a) and (4b) as proposed in [33]. We use the barrier method to solve the problem (1) as performed in [34]. In each step of this algorithm, the Newton's method is used as well.

The above mentioned solution has three disadvantages: 
(i) the constraints (1b) and (1c) are non-convex, (ii) the complexity is high, and (iii) it is central (not distributed). In the next section, a low complexity distributed solution is proposed for (1) based on FGs and message passing algorithms without the need for a central decision making mechanism.

\section{FACTOR-GRAPH BASED SOLUTION}

To develop a distributed algorithm, it is necessary to discriminate among a set of operations. These operations might be network agents, like D2D receivers and transmitters, and the action of cellular users. In order to reduce the complexity, one way is to break down the objective function and the constraints into a set of independent functions to solve the partial problems associated with these functions. Then, the partial solutions are exchanged to achieve the global solution. For this purpose, a graphical representation of functions and exchanged messages helps to visualize the general model of the network and to implement the MP algorithm [35]. Among graphical models, FGs work efficiently for decomposition of complex problems [24], [36]. An FG is basically a bipartite graph composed of variable nodes and factor nodes. The variable nodes represent the variables to be determined and the factor nodes represent the local functions serving as constraints and local decomposition of the main objective function. The connecting edges between these nodes represent a relationship between the variable nodes and their associated constraints of the optimization problem. An FG model is developed here to represent the proposed MP algorithm which contains relations between D2D receivers, transmitters and cellular users. In this model, QoS constraints on both cellular user and D2D pairs and the power allocation of each active user are considered.

Variable Nodes: Variable nodes are defined as $\left\{P_{j k}^{D}, P^{C}\right\}$ in which $P_{j k}^{D}$ indicates the power level of the $k$-th D2D transmitter linked to the receiver $j$. $P_{j k}^{D}$ should be determined with aiming to maximize the rate of each D2D pair, regarding (1c) and not to violate (1b) constraints as well. Furthermore, $P^{C}$ is the power level of co-channel cellular user to the eNB link that should be tracked by D2D receivers, for being able to adjust their transmit power level, see Layer-1 and Layer-3 in Fig. 2.

Factor Nodes: Considering (1), it is clear that (1a) implies a comparison between the rates of each active user to maximize the minimum level of each active user's rate. Therefore, factor nodes of the proposed FG are defined to coordinate the constraints of (1) and also to determine the rate of each user in the network. There are two types of factor nodes in this FG: (i) D2D transmitter factor nodes and (ii) D2D receiver factor nodes, denoted by $g_{j k}$ and $f_{j}$, respectively. D2D transmitter factor nodes $g_{j k}$ are responsible for QoS of D2D pairs and they coordinate (1e) in a D2D transmitter's terminal. However, factor nodes $f_{j}$ of D2D receivers are responsible for pairing constraints and QoS of cellular users (1d) in a D2D receiver's terminal. To meet (1c), $g_{j k}$ nodes are defined as follows:

$$
g_{j k}\left(P_{j k}^{D}\right)= \begin{cases}\log _{2}\left(1+\gamma_{j k}^{\prime D}\right) & \text { if } \gamma_{j k}^{\prime D} \geq \gamma_{\min }^{D}, P_{j k}^{D} \neq 0, \\ -\infty & \text { if } \gamma_{j k}^{\prime D}<\gamma_{\text {min }}^{D}, P_{j k}^{D} \neq 0, \\ 0 & \text { if } P_{j k}^{D}=0,\end{cases}
$$

where $\gamma_{j k}^{\prime D}$ is a simplified copy of SINR in D2D links (3), as follows:

$$
\begin{aligned}
& \gamma_{j k}^{\prime D}\left(P_{j k}^{D}\right)= \\
& \frac{P_{j k}^{D} h_{j k, j}^{2} d_{j k, j}^{-\alpha}}{P_{\max }^{C} h_{B, j}^{2} d_{B, j}^{-\alpha}+\sum_{\substack{j^{\prime}=1 \\
j^{\prime} \neq j}}^{N} \rho_{j^{\prime}} \sum_{k^{\prime}=1}^{L_{j}} \rho^{\prime} j^{\prime} k^{\prime} P_{\max }^{D} h_{j^{\prime} k^{\prime}, j}^{2} d_{j^{\prime} k^{\prime}, j}^{-\alpha}+N_{0}} .
\end{aligned}
$$

In which $P^{C}$ and $P_{j \prime k \prime}^{D}$ are replaced with $P_{\max }^{C}$ and $P_{\max }^{D}$, respectively. Similarly, to meet (1b), $f_{j}$ nodes are defined by:

$f_{j}\left(P^{C}, P_{j k}^{D}\right)= \begin{cases}\log _{2}\left(1+\gamma^{\prime C}\right) & \text { if } \sum_{k^{\prime}=1}^{L_{j}} \rho_{j k^{\prime}}^{\prime} \leq 1, \gamma^{\prime C} \geq \gamma_{\text {min }}^{C} \\ -\infty & \text { otherwise }\end{cases}$

where $\gamma^{\prime C}$ is the SINR of cellular link approximated by the $j$-th D2D receiver as:

$$
\frac{\gamma^{\prime C}\left(P^{C}, P_{j k}^{D}\right)=}{\rho_{j} \sum_{k=1}^{L_{j}} \rho_{j k} P_{j k}^{D} h_{j k, C}^{2} d_{j k, C}^{-\alpha}+\sum_{\substack{j^{\prime}=1 \\ j^{\prime} \neq j}}^{N} \rho_{j^{\prime}} \sum_{k=1}^{L_{j}} \rho \prime_{j^{\prime} k} P_{\max }^{D} h_{j^{\prime} k, C}^{2} d_{j^{\prime} k, C}^{-\alpha}+N_{0}} .
$$

By comparing (8) and (2), it is obvious that each D2D receiver assumes the worst case of network in which all other interfering users transmit the maximum allowed power. Moreover, the other assumptions could be derived from (5)(8). First, each D2D receiver adjusts its transmitter's pairing power level through the sent massages according to Fig. 2. Next, each D2D receiver would be paired with only one transmitter in an instance, where it does not have access to the other power allocation status of D2D links. Finally, we have to clarify the relations between the proposed FG and problem (1). In our problem, two consequent operations are minimum and maximum that operate on the rates of all active links in the network. Therefore, there is a comparison between D2D rates and the rates of cellular users to choose the minimum one, and accordingly this value should be maximized. Hence, we need to compare the calculated rates from the $g_{j k}$ nodes with the cellular rate calculated by the $f_{j}$ nodes. In Fig. 2, these two types of factor nodes are shown in Layer- 2 and Layer- 4 , respectively.

The Proposed MP Algorithm: A serial MP procedure exchanges the messages among the factor nodes and the variable nodes. It starts sending the messages from lower layer nodes towards upper layer ones and then returns the messages in backward direction. These messages are depicted by arrows in Fig. 2. Two types of messages are sent from factor nodes to variable nodes, depicted by solid arrows, and from variable nodes to factor nodes, depicted by dashed arrows. First, exchanging messages are initialized from Layer3 variable nodes towards Layer-4 and Layer-2 factor nodes. Next, the messages are sent out from Layer- 4 factor nodes towards Layer- 1 variable node. In the next step, the messages return in the opposite direction. The exchange of messages in the FG is not only a mathematical concept, but also there is a physical representation of message exchanging between D2D receivers and transmitters as well as among cellular user and receivers as a power assignment mechanism. The power 


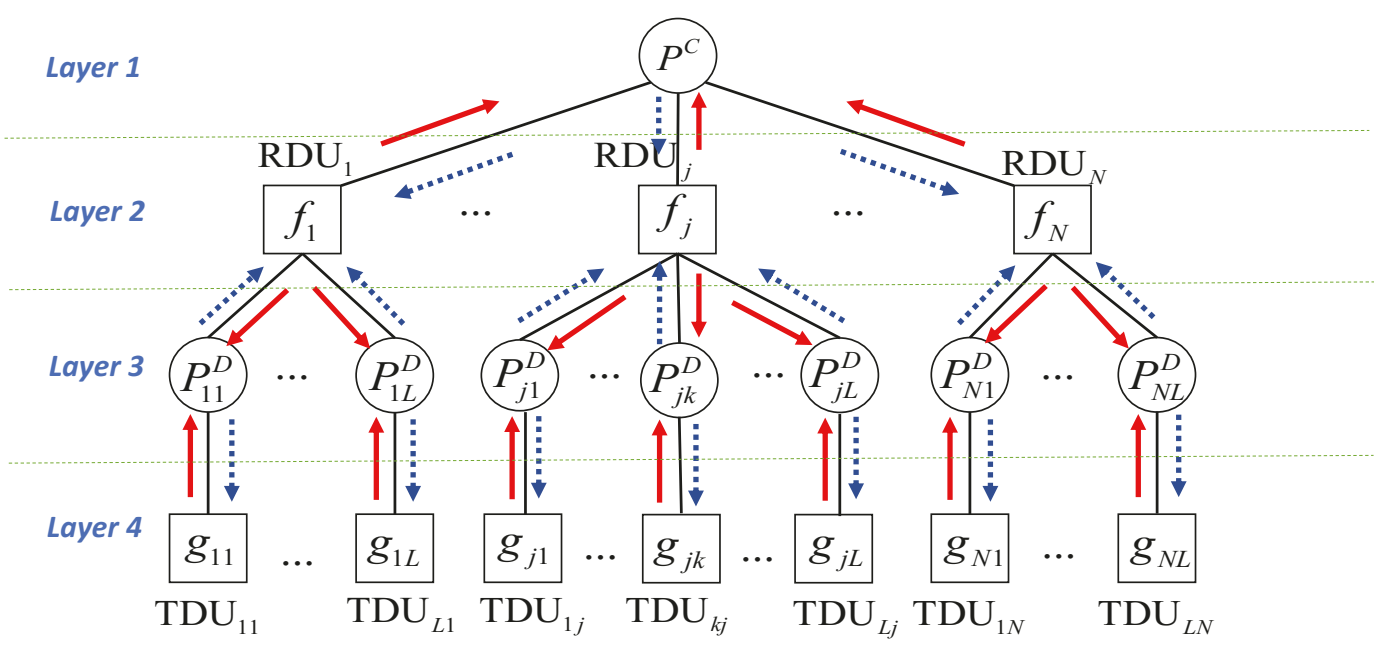

Fig. 2. In the proposed FG model, an RDU (a TDU) refers to Receiving (Transmitting) D2D User equipment. Two types of messages called factor-to-variable and variable-to-factor messages are shown with solid and dashed lines, respectively.

allocation usually affects each of network protocol stacks, including physical, MAC and network layer. However, we consider power assignment in the context of its effect on the interference of co-channel cellular and D2D users and this affects the MAC layer's functionality. Clearly, the MP is an iterative algorithm and the passing messages would converge to the final optimal solution after either termination of a predefined number $\ell_{\max }$ of iterations or an insignificant change in the achieved answers in successive iterations. Let $K$ be the number of quantized power levels associated to the power of cellular link and D2D links. Initial values of all the messages are zero. The factor-to-variable node messages are updated as follows:

$\boldsymbol{\mu}_{g_{j k} \rightarrow P_{j k}^{D}}^{(\ell)}=\max _{P_{j 1}^{D}, \ldots, P_{j L}^{D} \sim P_{j k}^{D}}\left(g_{j k}^{D}\left(P_{j k}^{D}\right)+\sum_{\substack{j^{\prime}=1 \\ j^{\prime} \neq j}}^{N} \sum_{\substack{k^{\prime}=1 \\ k^{\prime} \neq k}}^{L_{j}} \boldsymbol{\mu}_{P_{j^{\prime} k^{\prime}}^{D} \rightarrow g_{j k}}^{(\ell-1)}\right)$,

$$
\begin{array}{r}
\boldsymbol{\mu}_{f_{j} \rightarrow P^{C}}^{(\ell)}=\max _{P_{j 1}^{D}, \ldots, P_{j L}^{D} \sim P_{c}}\left(\operatorname { m i n } \left(f_{j}\left(P^{C}, P_{j 1}^{D}, \ldots, P_{j L}^{D}\right),\right.\right. \\
\left.\left.\sum_{k=1}^{L_{j}} \boldsymbol{\mu}_{g_{j k} \rightarrow P_{j k}^{D}}^{(\ell-1)}\right)\right) .
\end{array}
$$

Then, the calculated messages sent from Layer-1 variable node towards Layer-2 factor nodes are as follows:

$$
\boldsymbol{\mu}_{P^{C} \rightarrow f_{j}}^{(\ell)}=\max _{j^{\prime} \in\{1, \ldots, N\} \sim j}\left(\boldsymbol{\mu}_{f_{j^{\prime}} \rightarrow P^{C}}^{(\ell-1)}\right),
$$

and we finally have:

$$
\begin{array}{r}
\boldsymbol{\mu}_{f_{j} \rightarrow P_{j k}^{D}}^{(\ell)}=\max _{P_{j 1}^{D}, \ldots, P_{j L}^{D}, P^{C} \sim P_{j k}^{D}}\left(\operatorname { m i n } \left(f_{j}\left(P^{C}, P_{j 1}^{D}, \ldots, P_{j L}^{D}\right),\right.\right. \\
\left.\left.\boldsymbol{\mu}_{P^{C} \rightarrow f_{j}}^{(\ell)}+\sum_{\substack{k^{\prime}=1 \\
k^{\prime} \neq k}}^{L_{j}} \boldsymbol{\mu}_{g_{j k^{\prime}} \rightarrow P_{j k^{\prime}}^{D}}^{(\ell-1)}\right)\right) .
\end{array}
$$

By repeating (9)-(12), the value of $R_{0}$, which is equal to $R_{0 j}$, will be calculated as:

$$
R_{0}=\max _{0, \ldots, P_{\max }^{D}}\left(\boldsymbol{\mu}_{f_{j} \rightarrow P_{j k}^{D}}^{\left(\ell_{\max }\right)}\right) .
$$

By consideration of (9)-(13), it is understood that the MP algorithm breaks down the feasible solution area into smaller parts to achieve $R_{0}$. In each step, it compares the result of each part with the others to achieve the optimal point of (1a) in terms of all discrete values of variables $\left(P_{j k}^{D}\right.$ and $P^{C}$ ). Two consequent operations in Eq. (10) lead the result to maximize the minimum rates of active users. The second term of (10), i.e, the relation $\sum_{k=1}^{L_{j}} \boldsymbol{\mu}_{g_{j k} \rightarrow P_{j k}^{D}}^{(\ell-1)}$, refers to the pairing constraint which forces the receivers for being paired with one single transmitter in each receiver. In addition, a comparison is made among $N$ messages in Eq. (11) to choose the maximum value of the single rate of the cellular user. These messages are calculated by $N$ D2D receivers and the messages out from Layer-2 factor nodes towards Layer-3 variable nodes generated by Eq. (12). A simple pseudo-code of the proposed MP algorithm is presented in Algorithm 1.

\section{A. Sub-Optimality Analysis of the Approximated Solution}

In this part, the sub-optimal solution of the proposed MP algorithm is analyzed with simplified assumptions.

Proposition 1: The difference between an exact rate value $R^{C}$ for a cellular user and its approximated value $R^{\prime C}$ solution with the connection reduction, in order to avoid cycles in its graphical model, is as follows:

$$
R^{C}-R^{\prime C} \leq \log _{2}\left(1+\frac{(N-1)(K-1)}{N+K-1}\right) .
$$

proof: See Appendix A.

The upper bound of the difference between $R^{\prime C}$ and $R^{C}$ depends on the number of D2D pairs $N$ and the number of levels $K$ by which the users' power is quantized, instead of a continuous power selection. Obviously, by increasing $N$ and 


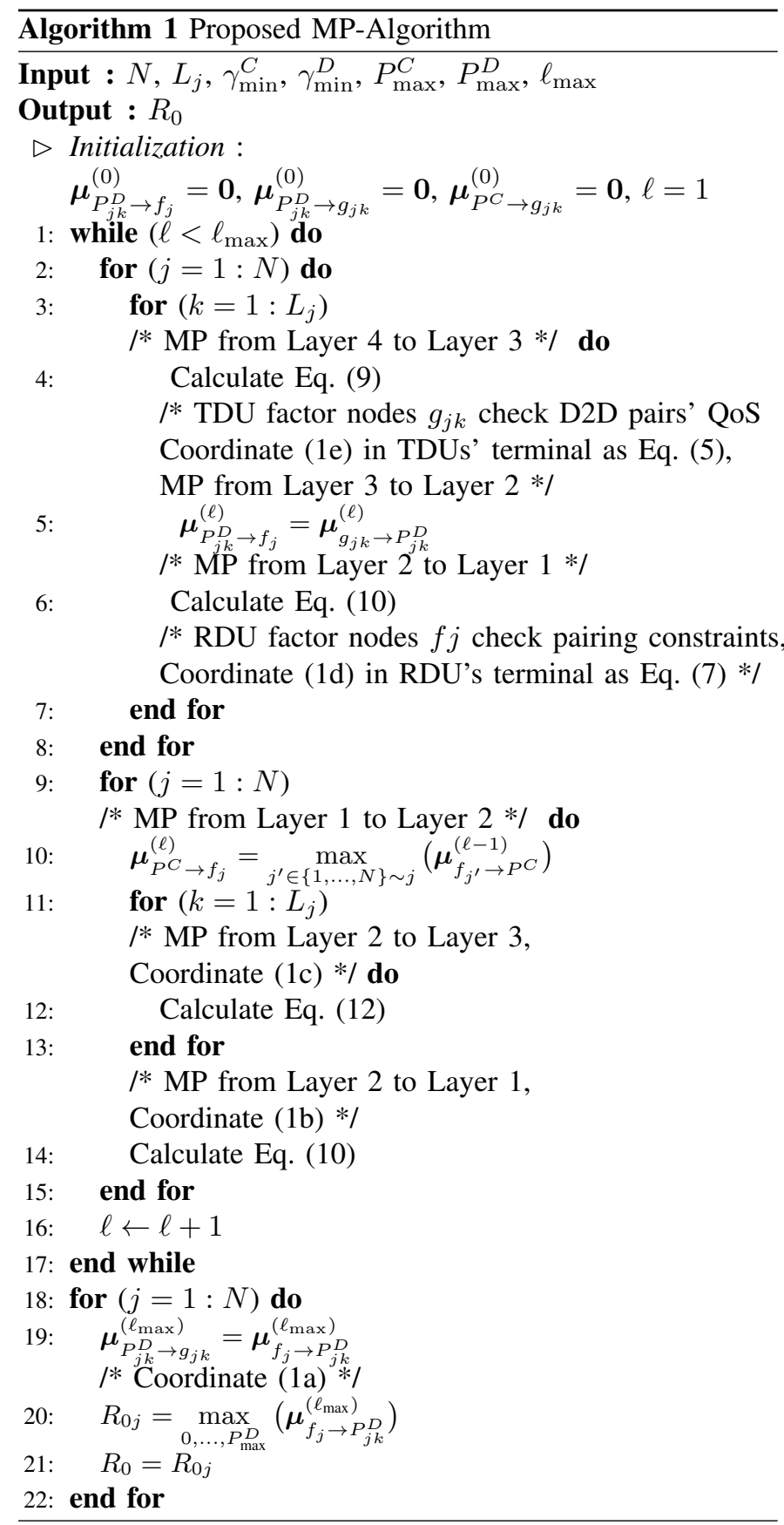

$K$, the approximation of $R^{C}$ degrades. However, we work around the limited values of $N$ and $K(N=4$ and $K=8$ at most), which forces a limited error to the calculations. Furthermore, the approximation rate converges to the true value of $R^{C}$ for $N=1$ due to vanishing the interference of other D2D pairs and the existence of only one cellular user and one D2D receiver in the FG model. Besides, for a small number of power levels, e.g., a binary power level, the approximation converges to a closer answer to the true value.

The difference between true and the MP approximated values of D2D rates, denoted respectively by $R_{j k}^{D}$ and $R_{j k}^{\prime} D$, is similarly given by:

$$
R_{j k}^{D}-R_{j k}^{\prime D} \leq \log _{2}\left(1+\frac{(N-1)(K-1)}{N+K-1}\right) .
$$

The inequality (15) presents that the difference between $R_{j k}^{D}$ and $R_{j k}^{\prime D}$ also depends on the number of power levels (scheduling options) $K$ and $N$. From (15) it can be easily seen that this difference increases monotonically, and converges to a limited value $\left(\log _{2}\left(1+\frac{(4-1)(8-1)}{4+8-1}\right) \approx 1.54 \mathrm{bits} / \mathrm{s} / H z\right)$ for $K=8$ and $N=4$.

The approximation of each user's rate in the max-min problem leads to a sub-optimal answer for $R_{0}$ and hence there is a difference from the optimal point. In this case, the discrepancy in $R_{0}$ is resulted from each user's approximation error of the rate. However, the approximation error in the maxsum problem would be accumulated and this is the main reason for the difference between analytical and simulation results, which will be seen in Section IV in more details.

\section{B. Analysis of the Converged Sum-Rate}

According to a large number of simulation results, we found that the converged point of the proposed MP algorithm can achieve better fairness results compared to the other objective functions like max-sum. A heuristic reason for that is the fact that the rate of each active user should be at least $R_{0}$ in the proposed MP algorithm. However, the sum-rate of the achieved point is less than the maximum of sum-rate among the possible solutions. To compare these cases, we have developed the maximization of sum-rate by using the max-sum MP algorithm [37], [38]. Thus, an FG model is applied to develop the maxsum MP algorithm similar to the one applied for the max-min MP algorithm in Fig. 2. For the max-sum case, the former Eqs. (10) and (12) become:

$$
\begin{gathered}
\boldsymbol{\mu}_{f_{j} \rightarrow P^{C}}^{(\ell)}=\max _{P_{j 1}^{D}, \ldots, P_{j L}^{D}}\left(\sum_{j=1}^{N} f_{j}\left(P^{C}, P_{j 1}^{D}, \ldots, P_{j L}^{D}\right), \sum_{k=1}^{L_{j}} \boldsymbol{\mu}_{g_{j k} \rightarrow P_{j k}^{D}}^{(\ell-1)}\right), \\
\boldsymbol{\mu}_{f_{j} \rightarrow P_{j k}^{D}}^{(\ell)}=\max _{P_{j 1}^{D}, \ldots, P_{j L}^{D}, P^{C} \sim P_{j k}^{D}}\left(f_{j}\left(P^{C}, P_{j 1}^{D}, \ldots, P_{j L}^{D}\right)+\right. \\
\boldsymbol{\mu}_{P^{C} \rightarrow f_{j}}^{(\ell)}+\sum_{\substack{k^{\prime}=1 \\
k^{\prime} \neq k}}^{L_{j}} \boldsymbol{\mu}_{\left.g_{j k^{\prime} \rightarrow P_{j k^{\prime}}}^{(\ell-1)}\right) .}
\end{gathered}
$$

Finally, the network sum-rate, denoted by $R^{t}$, is calculated by $\max _{0, \ldots, P_{\max }^{D}}\left(\boldsymbol{\mu}_{f_{j} \rightarrow P_{j k}^{D}}^{\left(\ell_{\max }\right)}\right)$

\section{Complexity}

In order to assess the complexity of the proposed method, assume that the number of neighboring factor nodes of Layer3 variable nodes is two (see Fig. 2) and denote the average number of neighboring variable nodes of Layer- 2 factor nodes by $L+1$. Moreover, the average number of the scheduling options of each variable node is denoted by $K$. By applying Eqs. (9)-(12), the complexity Layer-4 and Layer-2 factor nodes can be calculated as $\mathcal{O}(N L K)$ and $\mathcal{O}\left(N^{2} L^{2} K^{2}\right)$, respectively. Therefore, the total complexity of the proposed MP algorithm is in the order of $\mathcal{O}\left(N^{2} L^{2} K^{2}\right)$. On the contrary, the complexity of the exhaustive search for solving the suggested 
problem is $\mathcal{O}\left(N^{4} L^{N} \log (N L)\right)$, which is much more than that of the proposed method. The cost that is paid for this huge complexity reduction, is that the proposed method converges to a near optimal solution. The difference between the optimal solution with the achieved point arises from the simplified assumptions assumed in an FG to avoid cycles and extra exchanging messages among the users.

\section{Convergence}

Definition 1 (Coercive function) [39]: A function $g: \mathbb{R}^{n} \rightarrow$ $\mathbb{R}$ is termed coercive if, for every sequence of input variables $\left\{\mathbf{x}_{k}\right\} \subset \mathbb{R}^{n}$ with $\left\|\mathbf{x}_{k}\right\| \rightarrow \infty$, then $g\left(\mathbf{x}_{k}\right) \rightarrow \infty$. If $g($.$) is a$ coercive and continuous function, then a global minimum of $g($.$) must exist [39].$

Definition 2 (Pairwise separable convex program) [39]: An optimization problem is called pairwise separable convex program if its form is as follows:

$$
\begin{aligned}
\min F(\mathbf{x}) & =\sum_{i \in V} f_{i}\left(x_{i}\right)+\sum_{i, j \in E} f_{i j}\left(x_{i}, x_{j}\right), \\
\text { subject to } \quad \mathbf{x} & \in \mathbb{R}^{n},
\end{aligned}
$$

where $f_{i}($.$) factors are strictly convex, coercive, continuous,$ and $f_{i j}(.,$.$) factors are convex and twice continuously differ-$ entiable. The objective function $F($.$) is also strictly convex$ and coercive. Moreover, variables and edges of the associated FG of the problem are denoted by $V$ and $E$, respectively.

In order to use the results of [39] in the analysis of the convergence of the proposed MP algorithm, we modify our optimization problem to an unconstrained convex problem wherein the objective function is shown to be pairwise separable and the component functions are individually convex.

Proposition 2: There exists a modified unconstrained convex optimization problem associated to the optimization problem (1) through the Karush-Kuhn-Tucker (KKT) conditions.

Proof: See Appendix B.

Proposition 3: There exists a pairwise separable convex program for the proposed max-min optimization problem by which the updated equations of the max-min MP algorithm correspond to a convex problem converges to a unique optimal solution.

Proof: See Appendix C.

\section{E. Communication overhead}

In order to calculate the communication overhead, we calculate the vector size of messages passing from the lower layers of factor nodes to the upper layers of factor nodes, which is a physical message passing from D2D transmitter terminals to D2D receiver terminals. The vector size of each message passing from a TDU to an associated RDU linearly depends on $K$ (the number of possible power levels) in each link. To calculate the total size of messages received by each RDU, we have $L$ D2D transmitters for each of the $N$ D2D receivers. Thus, the vector size of passing messages in floating numbers (to store a floating-point number, 4-bytes (32 bits) memory will be allocated) would be $|L \times K|$ in the most dense case of our problem. It means that the overhead will be $(4 * 8 * 32$ bits $=0.1 \mathrm{KBs})$ in forward and backward directions when all the rates are optimized.

\section{SIMULATION RESULTS AND DISCUSSION}

For numerical analysis, we simulate a single cell with radius $500 \mathrm{~m}$, one cellular user and $N=1,2,3,4 \mathrm{D} 2 \mathrm{D}$ pairs which are allowed to share the resources of the cellular user, simultaneously. We also consider $L=2,3,4$ transmitters of D2D user (TDUs) for each receiver of D2D user (RDU) which could be paired by RDU. The maximum distance between the paired users is assumed to be $200 \mathrm{~m}$, the power of cellular (uplink) and D2D transmitters are assumed to be $0<P^{C}<P_{\max }$, $0<P_{j k}^{D}<P_{\max }$, respectively. In the following simulations, $P_{\max }$ is assumed $23 \mathrm{dBm}$ and the number of power levels $K$ associated to $P_{j k}^{D}$ is set to 8 . Moreover, the power of inactive D2D transmitters $P_{j k}^{D}$ is set to 0 . Small scale fading components of cellular-eNB link and D2D links is assumed to be Rayleigh faded with the path loss exponent $\alpha=3$ for all the links. At the beginning of each signal block or few blocks that corresponds to the qausi-static fading channel, the proposed MP algorithm runs on every node and it remains resistant to the link state changes. The MP convergence iteration limit is set to $\ell_{\max }=10$, while the convergence depends on the message initialization form. Since the flood initialization starts from all variables in Layer-4 simultaneously, the convergence would occur after one complete set of messages passed from Layer-4 to Layer-1 and returned to Layer-4. Each point in the numerical experiments was randomly repeated over 100 times by our own simulator developed in MATLAB software. In this section, the simulation results are presented in terms of maximized minimum rate for all active users, cellular and D2D sum-rate, network mean-rate and fairness level.

Moreover, the results of the proposed MP algorithm are compared with other works (e.g. [18], [19], [29], [38], [40], [41]) and centralized bench-marks such as exhaustive search results.

Proposed MP algorithm compared to other methods: In Fig. 3(a), the maximized minimum rate of all active users (D2D and cellular) applying the proposed MP algorithm is presented in terms of the number of TDUs for each RDU. It is shown that the achievable minimum rate of all active users decreases by increasing the number of RDUs due to the interference of D2D co-channel links. It also slightly increases by increasing the number of TDUs with a higher gradient for small number of users. In addition, the achieved sumrate of D2D pairs with the rate of a single cellular user is depicted in Figs. 3(b) and 3(c) in comparison with two other existing game-theoretic approaches [18], [19]. The scheme proposed in [18] is a Stackelberg game designed for D2D sum-rate maximization. Although the sum-rate of D2D pairs is maximized in [18], the share of the corresponding cellular user is low. Moreover, a hybrid-distributed scheme with the BS-supervision, proposed in [19], is depicted for two values of the maximum interference price denoted by $\theta^{\max }$. It is clear from Figs. 3(b) and 3(c) that the D2D pairs get much less share of the rate than the cellular user with a higher price and hence their QoS requirements might be violated. However, the performance of the scheme in [19] is quite close to our proposed scheme and the one in [18] with less pricing cost. It is also worthy to note that there is a trade-off between 
signaling overhead of MP algorithm on the one hand and on the other hand the learning delay and less precision of the achieved responses caused by establishing local information in the game-theoretic approaches, see e.g., [18], [19]. However, in the proposed scheme, we have simplified F G t o reduce the signaling overhead and also increase the precision of the achieved response, by sharing the local information among users to meet the advantages of both MP algorithm and noncooperative game-based approaches.

Max-Min vs. Max-Sum in mean-rate approach: The mean-rate of the network is studied in this example for the proposed MP algorithm. The results are compared with the ones of other studies like max-sum MP algorithm [29], [38], maxmin binary power control (BPC) exhaustive search, max-sum BPC exhaustive search [40], a suboptimal heuristic algorithm proposed in [41], two existing game-theoretic approaches [18], [19] and the extended dynamic target-SINR tracking power control (EDTPC) algorithm proposed in [11]. In the heuristic sub-optimal algorithm, for each active cellular user, a random feasible point is chosen for the binary vectors $\rho$ and $\rho_{j}^{\prime}$ and the initial sum-rate is computed by taking into account the binary vectors and the new values of $P^{C}, P^{D}$ extracted by using the max-min criterion in (1). Next, a neighbor point of binary vectors $\rho$ and $\rho_{j}^{\prime}$ is chosen and the optimization problem (1) is solved for each point and compared with the initial value of the sum-rate. The procedure is repeated to find the local maximum sum-rate of the network among the neighbor points. The results are depicted in Fig. 4 in terms of the number of co-channel D2D pairs with cellular user and 2 and 4 TDUs for each RDU. Clearly, the system mean-rate decreases by increasing the number of co-channel D2D pairs. Since the interference of D2D users imposed on the performance of cellular users is limited and QoS requirements provided by the cellular users are met, increasing the number of D2D pairs maximizes the spectral efficiency and consequently the network sum-rate. Moreover, increasing the number of TDUs for each RDU in order to make more choices, increases the network mean-rate as well. As a general rule, the achieved mean-rate of the proposed max-min MP algorithm is less than that of the max-sum MP algorithm and the max-sum BPC exhaustive search. However, it converges to the result of the max-sum algorithm by increasing the number of co-channel D2D pairs with a smaller slope compared to the max-sum algorithm. The reason is that the purpose of the max-sum problem is to maximize the sum-rate of the whole network and it does not concern individual users, while the max-min problem searches the best answer for the case all users' rates satisfy a minimum value constraint and that value would be maximized. Consequently, the performance of both algorithms leads to the same result by increasing the number of active users. On the other hand, the proposed MP algorithm achieves $90 \%$ of the max-min BPC exhaustive search performance in the worst case and about $97 \%$ in the best case by reducing the order of complexity from $\mathcal{O}\left(N^{4} L^{N} \log (N L)\right)$ to $\left.\mathcal{O}\left(N^{2} L^{2} K^{2}\right)\right)$. It almost achieved $100 \%$ of the max-min heuristics algorithm [41] in the best case and $92 \%$ in the worst case by reducing the order of complexity from $\mathcal{O}\left(N^{3} L^{3}\right)$ to $\mathcal{O}\left(N^{2} L^{2} K^{2}\right)$. Both of these compared algorithms are binary while the proposed
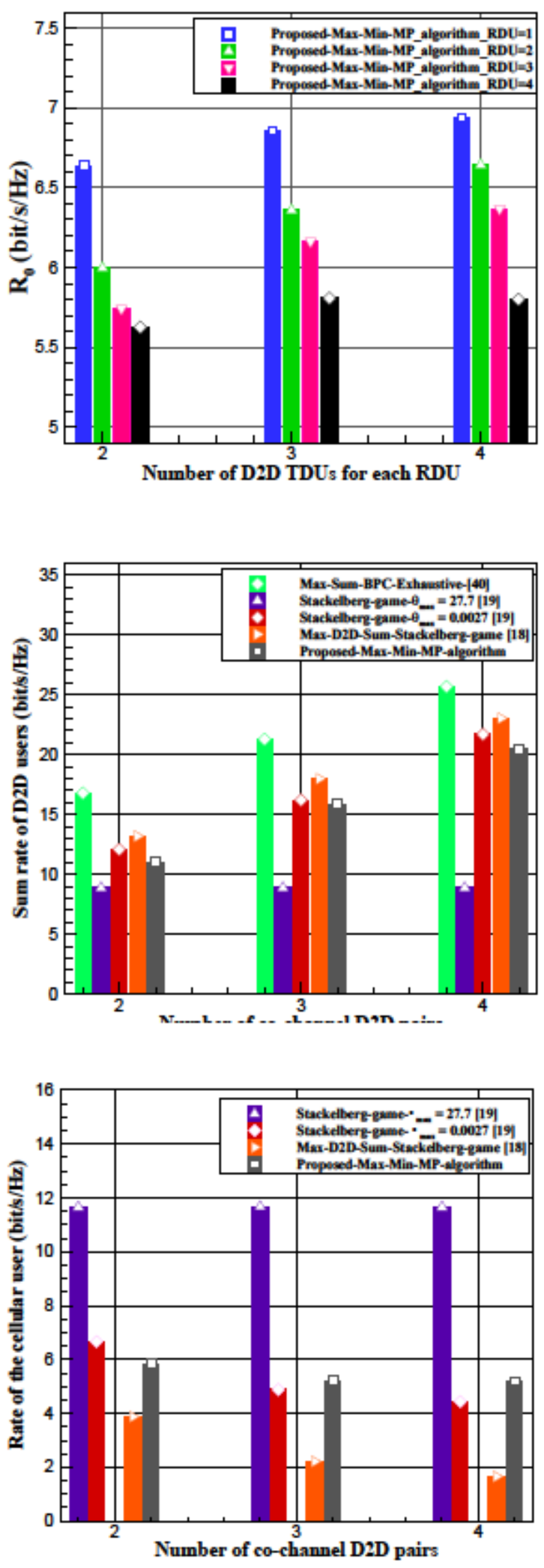

(c) Rate of the cellular user.

Fig. 3. Sum rates of D2D active links and a single cellular user for RDU values $2,3,4$, and $T D U=4$ and its comparisons with other studies.

algorithm works on $K$-ary values. Therefore, in the average $6.5 \%$ loss is negligible compared to the above mentioned 

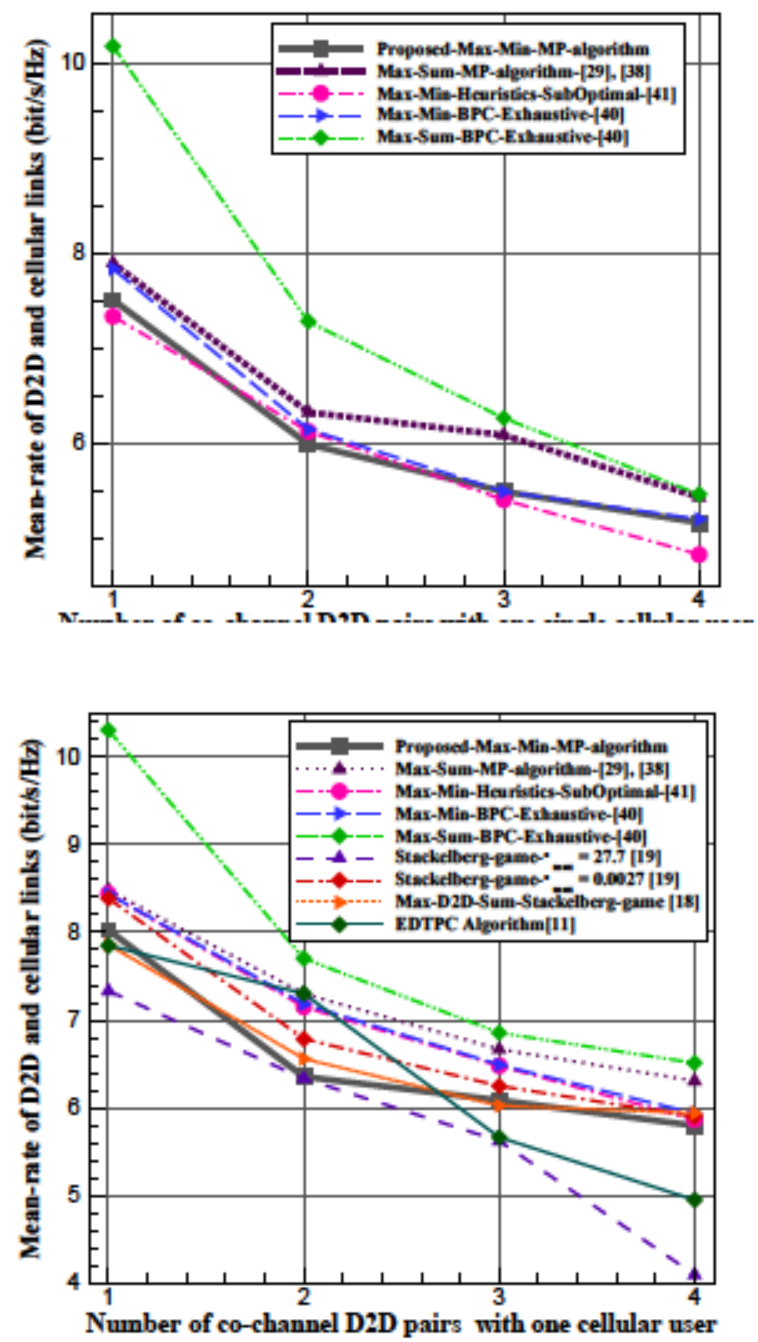

(b) For $\mathrm{TDU}=4$

Fig. 4. Mean-rate in terms of the number of co-channel $D 2 D$ pairs for $T D U=2$, 4 per RDU.

complexity reduction that the proposed algorithm attains in comparison with the centralized schemes. In comparison with the two other distributed game-theoretic schemes, it is clear that the performance of our proposed scheme is close to both works in [18] and [19]. However, the convergence of our proposed scheme is guaranteed and the power allocation is fair, compared to the two other schemes; these schemes try to keep the performance of some users near to the QoS level and this leads to achieving a Nash-equilibrium point which is far from the optimality. The other challenge of these schemes is the determination of the optimal pricing factor. The EDTPC algorithm proposed in [11] also has almost the same performance compared to the proposed max-min MP algorithm, while it is more sensitive to the number of cochannel users and minimum SINR threshold.

Max-Min vs. Max-Sum in fairness approach: Here, the fairness achieved by the proposed MP algorithm and the maxsum non-binary power control exhaustive search ( $K$ options) is investigated and the results are depicted in Fig. 5. In this experiment, the Jain's criterion is considered as the fairness metric, which converges to 1 in the most fair solution and to 0 in the worst fair point. The results are depicted in terms of mean-rate and different number of TDUs for each RDU in Figs. 5(a) and 5(b), respectively. It is clear that the higher fairness is obtained by the proposed MP algorithm compared to the one achieved by the non-binary exhaustive search in the max-sum problem. In Fig. 5(a), a monotonic relation is seen between fairness and mean-rate of the system. However, by increasing the number of active users, the system's meanrate decreases which adversely affects the system's sum-rate. Thus, the trade-off between the system's sum-rate and fairness is obvious in Fig. 5(a). On the other hand, the results confirm the fair performance of the proposed MP algorithm which varies in the range of 0.89 to 0.96 , specially for the higher number of RDUs. The fairness performance is better compared to the max-sum non-binary exhaustive search algorithm which is about $40 \%$ in the most different point and $28 \%$ in the least one. In Fig. 5(b), the difference between fairness of the max-min MP algorithm and the max-sum exhaustive search increases with increasing the number of potential TDUs. This result shows the effect of MP max-min algorithm in the context of fairly share of network resources between demanding D2D users. The individual rates of users are depicted in Fig. 6 for $L=4$ and $N=1,2,3,4$. The rates of active link achieved by the max-min MP algorithm are approximately equal, compared to the max-sum approach where the differences among rates of users are fairly high.

\section{CONCLUSION}

We have addressed joint D2D pairing and power allocation via a partially distributed scheme which does not need the full availability of the network information in the eNB. In the proposed method, a flexible and fair power allocation is formulated by utilizing the max-min criterion. A factorgraph model was developed to decompose the proposed maxmin optimization problem into multiple local functions. In the graphical representation of the problem, QoS constraints are included in the form of factor nodes and the power levels of transmitters are considered in form of variable nodes. Then, the distributed MP algorithm was proposed to solve the maximization of minimum rate for all active users, by exchanging messages between D2D receivers and transmitters and among receivers and cellular users, as well. The convergence of the proposed method to the optimal point was also proved in this paper. It was shown that the distributed MP algorithm can be employed for the optimization of joint power allocation and power control with considerably less complexity than the centralized methods. The proposed method is also much more precise in achieving the optimal point than partially distributed game-theoretic allocation methods. Moreover, the distributed MP algorithm presents an excellent performance in sum-rate maximization in underlay D2D networks without need for eNB signaling for power control with a negligible degradation in sum-rate. This degradation is only $6.5 \%$ in average compared with the sum-rate of benchmark schemes, e.g., max-min binary power control (BPC) exhaustive search. The proposed method can be applied in the recent release of the 

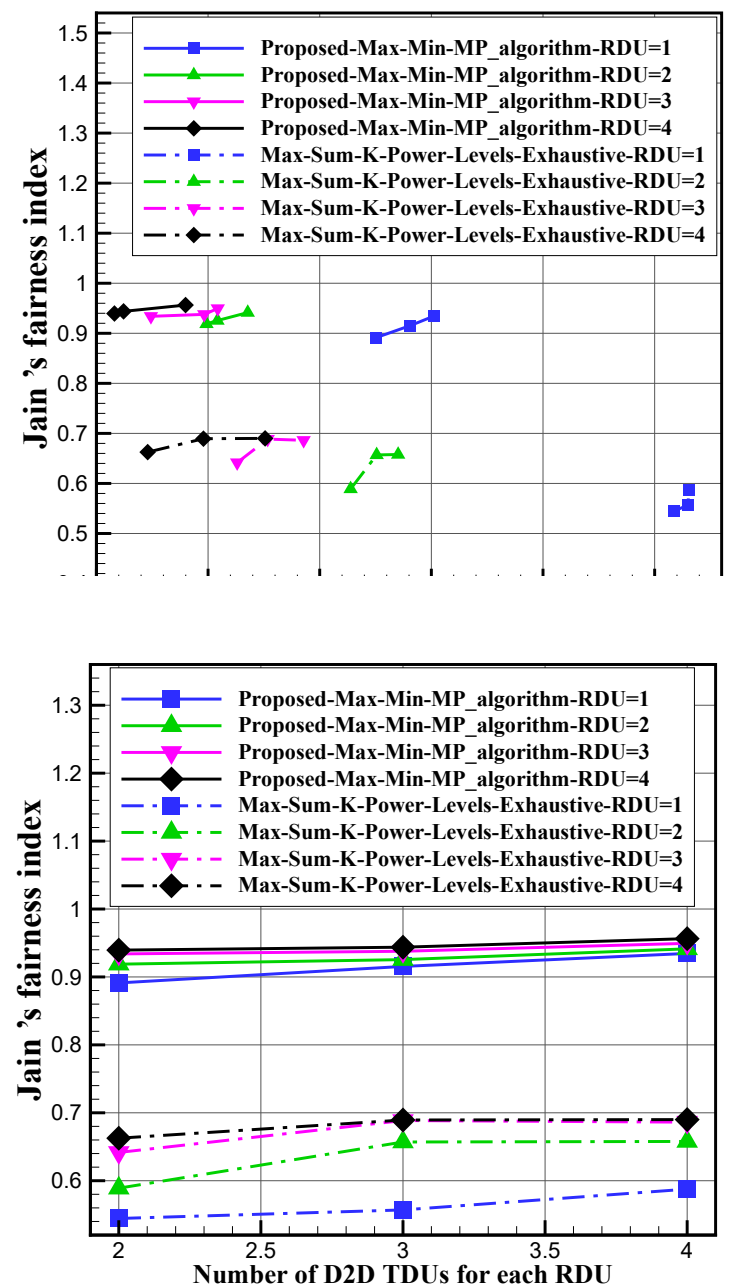

Fig. 5. Achieved fairness in terms of (a) mean-rate, (b) number of D2D TDUs.
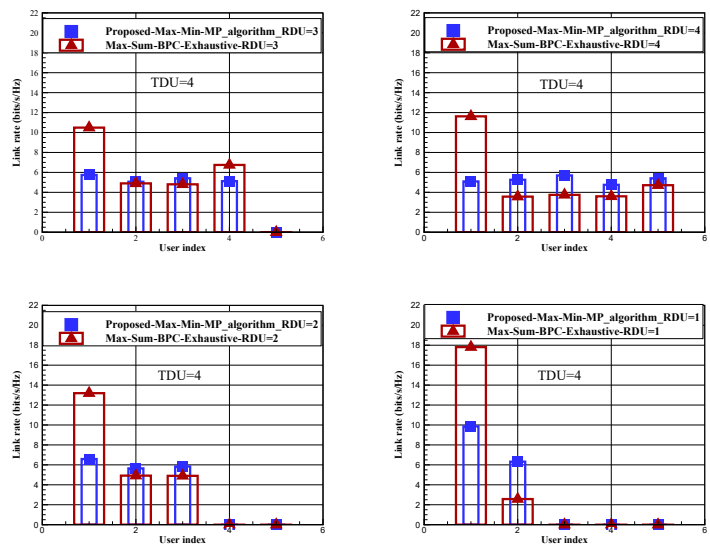

Fig. 6. Rates of D2D active links and a single cellular user for RDU values $1,2,3,4$, TDU $=4$ for max-min and max-sum approaches.

3GPP standard in which the controlling task of eNB might be diminished for power allocation of D2D users in highly dense networks. Besides, one of the most important applications of our proposed algorithm will be on Ultra-Reliable-LowLatency Communication (URLLC) networks. Since the power allocation and pairing of D2D communications on URLLC networks are challenging issues due to the explosive amount of devices in these networks, the future study can be applying the proposed MP algorithm on URLLC networks with applications in $5 \mathrm{G}$ wireless communication and beyond networks.

\section{APPENDIX A \\ PROOF OF PROPOSITION 1}

In order to find the difference between the exact solution and the approximated one with simplified assumptions, we calculate the range of error occurred in terms of different power scheduling. We know that $\gamma^{C} \gg 1$, therefore, the difference is calculated as shown in the equation set (18).

In (18), if we assume the worst case that our guess has been completely wrong for all other D2D users where they were still active $\left(P_{j^{\prime} k}^{D} \neq 0\right)$, we will have $P_{j^{\prime} k}^{D}=\frac{P_{\max }^{D}}{K}$ which is the minimum power level that can be assumed for an active user. Therefore, it would maximize the numerator and minimize the second summation of the denominator. Moreover, we know the exact value of the first term of the denominator, since it is the power of the under investigation D2D user. If we set $P_{j^{\prime} k}^{D}=P_{\max }^{D}$, the difference would be in the form of (19). Similarly, the difference is calculated for D2D rates as demonstrated in the equation set (19).

The achieved approximation of the equation set (19) with the assumption of $P_{j^{\prime} k}^{D}=\frac{P_{\max }^{D}}{K}$ and $P^{C}=P_{\max }^{C}$ would be as the last line of (19) where all other D2D pairs are active with the minimum power level.

\section{APPENDIX B \\ PROOF OF PROPOSITION 2}

In order to transform the problem (1) to an equivalent unconstrained problem, the following steps are required. Generally, if we have the optimization problem in the form of (20)

$$
\begin{cases}\text { minimize } & F(\mathbf{x}), \\ \text { sub. to } & g_{i}(\mathbf{x}) \leq 0, i \in \mathcal{C},\end{cases}
$$

where $\mathcal{C}$ denotes the index set of constraints, the Lagrange setup for this problem is written as $L(\mathbf{x}, \lambda) \triangleq F(\mathbf{x})+$ $\sum_{i \in \mathcal{C}} \lambda_{i} g_{i}(\mathbf{x})$, where $\mathbf{x}$ and $\lambda_{i}$ denote the optimization variable and the KKT multipliers, respectively. By considering inequality constraints, the KKT approach generalizes the method of Lagrange multipliers to the nonlinear programming, which allows only equality constraints. If $x^{*}$ is a local optimum for the mentioned optimization problem, then there exist constants like $\lambda_{i}^{*}, i \in \mathcal{C}$, called KKT multipliers, such that

$$
\begin{aligned}
& \nabla F\left(x^{*}\right)=\sum_{i \in \mathcal{C}} \lambda_{i}^{*} \nabla g_{i}\left(x^{*}\right), \\
& g_{i}\left(\mathbf{x}^{*}\right) \leq 0, \quad i \in \mathcal{C}, \\
& \lambda_{i}^{*} \geq 0, \\
& \lambda_{i}^{*} g_{i}\left(\mathbf{x}^{*}\right)=0, \quad i \in \mathcal{C} .
\end{aligned}
$$




$$
\begin{aligned}
& R^{C}-R^{\prime C}=\log _{2}\left(1+\frac{P^{C} h_{B, C}^{2} d_{B, C}^{-\alpha}}{\sum_{j=1}^{N} \rho_{j} \sum_{k=1}^{L_{j}} \rho^{\prime}{ }_{j k} P_{j k}^{D} h_{j k, C}^{2} d_{j k, C}^{-\alpha}+N_{0}}\right) \\
& -\log _{2}\left(\frac{P^{C} h_{B, C}^{2} d_{B, C}^{-\alpha}}{\rho_{j} \sum_{k=1}^{L_{j}} \rho_{j k}^{\prime} P_{j k}^{D} h_{j k, C}^{2} d_{j k, C}^{-\alpha}+\sum_{\substack{j^{\prime}=1 \\
j^{\prime} \neq j}}^{N} \rho_{j^{\prime}} \sum_{k=1}^{L_{j}} \rho_{j^{\prime} k}^{\prime} P_{\max }^{D} h_{j^{\prime} k, C}^{2} d_{j^{\prime} k, C}^{-\alpha}+N_{0}}\right) \\
& \rho_{j} \sum_{k=1}^{L_{j}} \rho_{j k}^{\prime} P_{j k}^{D} h_{j k, C}^{2} d_{j k, C}^{-\alpha}+\sum_{j^{\prime}=1}^{N} \rho_{j^{\prime}} \sum_{k=1}^{L_{j}} \rho_{j^{\prime} k}^{\prime} P_{\max }^{D} h_{j^{\prime} k, C}^{2} d_{j^{\prime} k, C}^{-\alpha}+N_{0} \\
& \approx \log _{2}\left(\frac{c^{\prime} \neq j}{\sum_{j=1}^{N} \rho_{j} \sum_{k=1}^{L_{j}} \rho^{\prime}{ }_{j k} P_{j k}^{D} h_{j k, C}^{2} d_{j k, C}^{-\alpha}+N_{0}}\right. \\
& =\log _{2}\left(1+\frac{\sum_{\substack{j^{\prime}=1 \\
j^{\prime} \neq j}}^{N} \rho_{j^{\prime}} \sum_{k=1}^{L_{j}} \rho_{j^{\prime} k}^{\prime}\left(P_{\max }^{D}-P_{j^{\prime} k}^{D}\right) h_{j^{\prime} k, C}^{2} d_{j^{\prime} k, C}^{-\alpha}}{\rho_{j} \sum_{k=1}^{L_{j}} \rho_{j k}{ }_{j k} P_{j k}^{D} h_{j k, C}^{2} d_{j k, C}^{-\alpha}+\sum_{\substack{j^{\prime}=1 \\
j^{\prime} \neq j}}^{N} \rho_{j^{\prime}} \sum_{k=1}^{L_{j}} \rho_{j^{\prime} k}^{\prime} P_{j^{\prime} k}^{D} h_{j^{\prime} k, C}^{2} d_{j^{\prime} k, C}^{-\alpha}+N_{0}}\right) \\
& \sum_{j^{\prime}=1}^{N} \rho_{j^{\prime}} \sum_{k=1}^{L_{j}} \rho_{j^{\prime} k}^{\prime} P_{\max }^{D}\left(\frac{K-1}{K}\right) h_{j^{\prime} k, C}^{2} d_{j^{\prime} k, C}^{-\alpha} \\
& \leq \log _{2}\left(1+\frac{\substack{j^{\prime}=1 \\
j^{\prime} \neq j}}{\rho_{j} \sum_{k=1}^{L_{j}} \rho^{\prime}{ }_{j k} P_{\max }^{D} h_{j k, C}^{2} d_{j k, C}^{-\alpha}+\sum_{\substack{j^{\prime}=1 \\
j^{\prime} \neq j}}^{N} \rho_{j^{\prime}} \sum_{k=1}^{L_{j}} \rho_{j^{\prime} k}^{\prime} P_{\max }^{D}\left(\frac{1}{K}\right) h_{j^{\prime} k, C}^{2} d_{j^{\prime} k, C}^{-\alpha}+N_{0}}\right) \\
& \leq \log _{2}\left(1+\frac{(N-1) P_{\max }^{D}\left(\frac{K-1}{K}\right)}{P_{\max }\left(\frac{N+K-1}{K}\right)}\right)=\log _{2}\left(1+\frac{(N-1)(K-1)}{N+K-1}\right) .
\end{aligned}
$$

$$
\begin{aligned}
& R_{j k}^{D}-R_{j k}^{\prime D}=\log _{2}\left(1+\frac{P_{j k}^{D} h_{j k, j}^{2} d_{j k, j}^{-\alpha}}{P^{C} h_{B, j}^{2} d_{B, j}^{-\alpha}+\sum_{\substack{j^{\prime}=1 \\
j^{\prime} \neq j}}^{N} \rho_{j^{\prime}} \sum_{\substack{k^{\prime}=1 \\
k^{\prime} \neq k}}^{L_{j}} \rho_{j^{\prime} k^{\prime}}^{\prime} P_{j^{\prime} k^{\prime}}^{D} h_{j^{\prime} k^{\prime}, j}^{2} d_{j^{\prime} k^{\prime}, j}^{-\alpha}+N_{0}}\right) \\
& -\log _{2}\left(\frac{P_{j k}^{D} h_{j k, j}^{2} d_{j k, j}^{-\alpha}}{P_{\max }^{C} h_{B, j}^{2} d_{B, j}^{-\alpha}+\sum_{\substack{j^{\prime}=1 \\
j^{\prime} \neq j}}^{N} \rho_{j^{\prime}} \sum_{\substack{k^{\prime}=1 \\
k^{\prime} \neq k}}^{L_{j}} \rho_{j^{\prime} k^{\prime}}^{\prime} P_{\max }^{D} h_{j^{\prime} k^{\prime}, j}^{2} d_{j^{\prime} k^{\prime}, j}^{-\alpha}+N_{0}}\right) \\
& \approx \log _{2}\left(1+\frac{\left(P_{\max }^{C}-P^{C}\right) h_{B, j}^{2} d_{B, j}^{-\alpha}+\sum_{\substack{j^{\prime}=1 \\
j^{\prime} \neq j}}^{N} \rho_{j^{\prime}} \sum_{\substack{k^{\prime}=1 \\
k^{\prime} \neq k}}^{L_{j}} \rho_{j^{\prime} k^{\prime}}^{\prime}\left(P_{\max }^{D}-P_{j^{\prime} k^{\prime}}^{D}\right) h_{j^{\prime} k^{\prime}, j}^{2} d_{j^{\prime} k^{\prime}, j}^{-\alpha}}{P^{C} h_{B, j}^{2} d_{B, j}^{-\alpha}+\sum_{\substack{j^{\prime}=1 \\
j^{\prime} \neq j}}^{N} \rho_{j^{\prime}} \sum_{\substack{k^{\prime}=1 \\
k^{\prime} \neq k}}^{L_{j}} \rho_{j^{\prime} k^{\prime}}^{\prime} P_{j^{\prime} k^{\prime}}^{D} h_{j^{\prime} k^{\prime}, j}^{2} d_{j^{\prime} k^{\prime}, j}^{-\alpha}+N_{0}}\right) . \\
& \left(P_{\max }^{C}-P_{\max }^{C}\right) h_{B, j}^{2} d_{B, j}^{-\alpha}+\sum_{j^{\prime}=1}^{N} \rho_{j^{\prime}} \sum_{k^{\prime}=1}^{L_{j}} \rho_{j^{\prime} k^{\prime}}^{\prime}\left(P_{\max }^{D}-\frac{P_{\max }^{D}}{K}\right) h_{j^{\prime} k^{\prime}, j}^{2} d_{j^{\prime} k^{\prime}, j}^{-\alpha} \\
& \leq \log _{2}\left(1+\frac{\substack{j^{\prime}=1 \\
j^{\prime} \neq j}}{P_{\max }^{C} h_{B, j}^{2} d_{B, j}^{-\alpha}+\sum_{\substack{j^{\prime}=1 \\
j^{\prime} \neq j}}^{N} \rho_{j^{\prime}} \sum_{\substack{k^{\prime}=1 \\
k^{\prime} \neq k}}^{L_{j}} \rho^{\prime} j_{j^{\prime} k^{\prime}}^{\prime} \frac{P_{\max }^{D}}{K} h_{j^{\prime} k^{\prime}, j}^{2} d_{j^{\prime} k^{\prime}, j}^{-\alpha}}\right) \leq \log _{2}\left(1+\frac{(K-1)(N-1)}{K+N-1}\right) .
\end{aligned}
$$


where (21a)-(21d) denotes respectively the stationarity, primal feasibility, dual feasibility, and complementary slackness conditions. These first-order conditions k nown as the K KT conditions are satisfied at the stationary points of the Lagrangian setup. They are necessary conditions for the optimum of a constrained problem. However, the first-order c onditions are not sufficient to g uarantee a local minimum. To provide this, we remark the second-order sufficient conditions. The optimal point which satisfies the KKT necessary conditions of the optimality at $x^{*}$, will be a global optimal point for the nonlinear optimization problem, as long as the given Hessian matrix of Lagrangian, is positive definite, i .e., $\boldsymbol{z}^{\mathrm{T}} \nabla_{R_{k}}^{2} L\left(\mathbf{R}^{*}, \lambda^{*}\right) \boldsymbol{z}$. Furthermore, $\boldsymbol{z}$ should span the null-space of the matrix of the gradient of active constraints at the optimal points [34]. Following the problem (1), an approximation of minimizing operator is introduced at first as follows:

$$
R_{0} \triangleq-\frac{1}{\xi} \log \left(\frac{1}{N} \sum_{i=1}^{N} e^{-\xi R_{i}}\right),
$$

where $\xi$ is a constant which is $\xi \gg 1$, and $N$ denotes the number of active users and $R_{i}$, for $1 \leq i \leq N$, is the rate of the $i$-th active user. Thus, (1b) and (1c) constraints will be respectively as follows:

$$
\begin{aligned}
& \log \left(1+\gamma^{C}\right) \geq-\frac{1}{\xi} \log \left(\frac{1}{N} \sum_{i=1}^{N} e^{-\xi R_{i}}\right), \forall j \in \mathcal{D}^{r}, \forall k \in \mathcal{D}^{t}, \\
& \log \left(1+\gamma_{j k}^{D}\right) \geq-\frac{1}{\xi} \log \left(\frac{1}{N} \sum_{i=1}^{N} e^{-\xi R_{i}}\right), \forall j \in \mathcal{D}^{r}, \forall k \in \mathcal{D}^{t} .
\end{aligned}
$$

Accordingly, (1b) and (1c) clearly could be omitted from the problem since for $\xi \rightarrow \infty$, the approximation function converges to the minimum amount, and hence it would be satisfied for each $R_{i}, 1 \leq i \leq N$, as $R_{i} \geq-\frac{1}{\xi} \log \left(\frac{1}{N} \sum_{i=1}^{N} e^{-\xi R_{i}}\right)$. In this case, (1d) and (1e) would be as following:

$$
\begin{aligned}
& \frac{1}{\xi} \log \left(\frac{1}{N} \sum_{i=1}^{N} e^{-\xi R_{i}}\right)+\log \left(1+\gamma_{\text {min }}^{C}\right) \leq 0, \\
& \frac{1}{\xi} \log \left(\frac{1}{N} \sum_{i=1}^{N} e^{-\xi R_{i}}\right)+\log \left(1+\gamma_{\text {min }}^{D}\right) \leq 0 .
\end{aligned}
$$

Next, the necessary optimality conditions are specified through the use of Lagrangian function which is given by:

$$
\begin{aligned}
& L(R, \lambda) \triangleq \frac{1}{\xi} \log \left(\frac{1}{N} \sum_{i=1}^{N} e^{-\xi R_{i}}\right) \\
+ & \lambda_{1}\left(\frac{1}{\xi} \log \left(\frac{1}{N} \sum_{i=1}^{N} e^{-\xi R_{i}}\right)+\log \left(1+\max \left(\gamma_{\min ^{C}}, \gamma_{\min ^{D}}\right)\right)\right) .
\end{aligned}
$$

Hence, we have:

$$
\begin{aligned}
L(R, \lambda) & =\frac{1}{\xi} \log \left(\frac{1}{N} \sum_{i=1}^{N} e^{-\xi R_{i}}\right) \\
+ & \lambda_{1}\left(\frac{1}{\xi} \log \left(\frac{1}{N} \sum_{i=1}^{N} e^{-\xi R_{i}}\right)+\max \left(R_{\min }^{C}, R_{\min }^{D}\right)\right) .
\end{aligned}
$$

To achieve the KKT multipliers from the first KKT condition, the gradient of our objective Lagrangian is calculated as follows:

$$
\nabla L(R, \lambda)=\left\{\begin{array}{c}
-\left(\frac{e^{-\xi R_{1}}}{\sum_{i=1}^{N} e^{-\xi R_{i}}}\right)+\lambda_{1}\left(\frac{e^{-\xi R_{1}}}{\sum_{i=1}^{N} e^{-\xi R_{i}}}\right)=0, \\
\vdots \\
-\left(\frac{e^{-\xi R_{N}}}{\sum_{i=1}^{N} e^{-\xi R_{i}}}\right)+\lambda_{1}\left(\frac{e^{-\xi R_{N}}}{\sum_{i=1}^{N} e^{-\xi R_{i}}}\right)=0,
\end{array}\right.
$$

Hence, we have $\lambda_{1}=\lambda_{1}^{*}$. The complementary slackness condition is also satisfied as follows:

$\lambda_{1}^{*}\left(\frac{1}{\xi} \log \left(\frac{1}{N} \sum_{i=1}^{N} e^{-\xi R_{i}}\right)+\log \left(1+\max \left(\gamma_{\min }^{C}, \gamma_{\min }^{D}\right)\right)\right)=0$, which results in $\mathbf{R}^{*}=\left(R_{1}^{c^{*}}, R_{1}^{d^{*}}, R_{2}^{d^{*}}, \ldots ., R_{N}^{d^{*}}\right)^{\mathrm{T}}$.

In general, the KKT conditions can be satisfied at a local optimum, a global optimum (solution of the problem) as well as at a saddle point. Here, we used the KKT conditions to characterize all the stationary points of the problem, and then we should perform some additional testing (second-order conditions) to determine the optimal solutions of the problem (global optimum of the constrained problem). Hence, we have:

$$
\nabla_{R_{i}}^{2} L(\mathbf{R}, \lambda)=\left(\frac{1}{\mathbf{1}^{\mathrm{T}} \mathbf{r}} \operatorname{diag}(\mathbf{r})-\frac{1}{\left(\mathbf{1}^{\mathrm{T}} \mathbf{r}\right)^{2}} \mathbf{r r}^{\mathrm{T}}\right)\left(1+\lambda_{1}\right),
$$

where $\mathbf{1}$ is a column-wise all-one vector, $\mathbf{r}=$ $\left(e^{-\xi R_{1}}, \ldots, e^{-\xi R_{N}}\right)^{\mathrm{T}}$ and

$$
\operatorname{diag}(\mathrm{r})=\left(\begin{array}{cccc}
e^{-\xi R_{1}} & 0 & \ldots & 0 \\
\vdots & \ldots & \ddots & \vdots \\
0 & \ldots & 0 & e^{-\xi R_{N}}
\end{array}\right) .
$$

To find the null-space of the gradient of active constraints, the gradient of active constraints are as follows:

$$
\mathbf{A}^{*}=\left(\begin{array}{cc}
\frac{-e^{-\xi R_{1}^{d^{*}}}}{\sum_{i=1}^{N} e^{-\xi R_{i}^{d^{*}}}} & \frac{-e^{-\xi R_{1}^{c^{*}}}}{\sum_{i=1}^{N} e^{-\xi R_{i}^{c^{*}}}} \\
\vdots & \vdots \\
\frac{-e^{-\xi R_{N}^{d^{*}}}}{\sum_{i=1}^{N} e^{-\xi R_{i}^{d^{*}}}} & \frac{-e^{-\xi R_{N}^{c^{*}}}}{\sum_{i=1}^{N} e^{-\xi R_{i}^{c^{*}}}}
\end{array}\right)^{T},
$$

where the echelon form of $\mathbf{A}^{*}$ is as follows:

$$
\mathbf{E}_{\mathbf{A}^{*}}=\left(\begin{array}{ccc}
e^{-\xi R_{1}^{d^{*}}}-e^{-\xi R_{1}^{c^{*}}} & \ldots & e^{-\xi R_{N}^{d^{*}}}-e^{-\xi R_{N}^{c^{*}}} \\
0 & \ldots & 0
\end{array}\right) .
$$

Hence, the null-space equation $\mathbf{E}_{\mathbf{A} * \mathbf{Z}}=\mathbf{0}$ results in $\sum_{i=1}^{N}\left(e^{-\xi R_{i}^{d^{*}}}-e^{-\xi R_{i}^{c^{*}}}\right) R_{i}=0$. Let $b_{i} \triangleq e^{-\xi R_{i}^{d^{*}}}-e^{-\xi R_{i}^{c^{*}}}$, then we have:

$$
\mathbf{z} \in \mathcal{N}\left(\mathbf{A}^{*}\right)=\left(\begin{array}{cccc}
\frac{b_{2}}{b_{1}} & \frac{b_{2}}{b_{1}} & \ldots & \frac{b_{N}}{b_{1}} \\
1 & 0 & \ldots & 0 \\
\vdots & \ddots & \ldots & \vdots \\
0 & 0 & \ldots & 1
\end{array}\right)
$$

where $\mathcal{N}\left(\mathbf{A}^{*}\right)$ indicates the null-space of $\mathbf{A}^{*}$. Now, we must verify that for all $\boldsymbol{z} \in \mathcal{N}\left(\mathbf{A}^{*}\right), \boldsymbol{z}^{T} \nabla_{R_{i}}^{2} L\left(\mathbf{R}^{*}, \lambda^{*}\right) \boldsymbol{z}$ is positive. 
By some manipulations, we have:

$$
\begin{aligned}
& \boldsymbol{z} \nabla_{R_{i}}^{2} L(\mathbf{R}, \lambda) \boldsymbol{z}^{T}= \\
& \quad\left(\frac{\sum_{i=1}^{N} z_{i}^{2} R_{i} \sum_{i=1}^{N} R_{i}}{\left(\sum_{i=1}^{N} R_{i}\right)^{2}}-\frac{\left(\sum_{i=1}^{N} z_{i} R_{i}\right)^{2}}{\left(\sum_{i=1}^{N} R_{i}\right)^{2}}\right) \lambda_{1}^{*} \stackrel{(a)}{>} 0
\end{aligned}
$$

where $(a)$ is obtained from the Cauchy-Schwarz inequality, and hence the proof is complete.

\section{APPENDIX C}

\section{PROOF OF PROPOSITION 3}

In the proposed max-min algorithm, the problem could be modified to the form of:

$$
\begin{aligned}
\max \quad F(\mathbf{R}) & =\min \left(R_{1}^{C}, R_{1}^{D}, \ldots, R_{N}^{D}\right)\left(1+\lambda^{*}\right) \\
& -\log \left(1+\max \left(\gamma_{\min }^{C}, \gamma_{\min }^{D}\right)\right) .
\end{aligned}
$$

We need to show that this form of the proposed problem corresponds to a separable convex program. Thus, we need to prove that $-F(\mathbf{R})$ is convex as well as separable to the convex, coercive, and twice differentiable factors. First, we need to prove the convexity of function $-F(\mathbf{R})$ by calculating

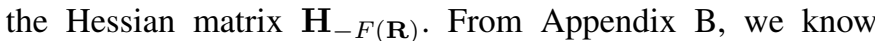
that the Hessian of log-sum-exp function, i.e., $-F(\mathbf{R})=$ $\frac{1}{\xi} \log \left(\frac{1}{N} \sum_{i=1}^{N} e^{-\xi R_{i}}\right)$, would be in the form of

$$
\nabla_{R_{i}}^{2}(-F(\mathbf{R}))=\left(\frac{1}{\mathbf{1}^{T} \boldsymbol{r}} \operatorname{diag}(\mathbf{r})-\frac{1}{\left(\mathbf{1}^{T} \mathbf{r}\right)^{2}} \mathbf{r r}^{T}\right)
$$

To show that $\nabla_{R_{i}}^{2}(-F(\mathbf{R}))$ is positive definite, we must verify that $\boldsymbol{v}^{T} \nabla_{R_{i}}^{2}(-F(\mathbf{R})) \boldsymbol{v} \geq 0$ for all vectors $\boldsymbol{v}$. This is clearly approved by using Cauchy-Schwartz inequality (see Appendix B). Next, we should check the separability of $F(\mathbf{R})$ to the convex factors. By referring to the proposed FG model (see Fig. 2) we have a cycle-free FG and the minimum operator has the associative property. Therefore, the $F(\mathbf{R})$ function could be separated to the functions of the lower layer (Layer-4) and the upper layer (Layer-2) factor nodes. At the next step, we have to verify the convexity of $f_{j}\left(P_{j 1}^{d}, \ldots, P_{j L}^{D}\right)$ and $g_{j k}^{D}\left(P_{j k}^{D}\right)$ functions as the factor nodes of each layer. By the same way mentioned in (4a) and (4b), the rate of D2D users and cellular ones would be, respectively, approximated as following, for $\gamma_{j k}^{\prime D}, \gamma^{\prime C} \gg 1$,

$$
\begin{aligned}
& R_{j k}^{D} \simeq \log _{2}\left(\gamma_{j k}^{\prime} D\left(P_{j k}^{D}\right)\right)= \\
& \log _{2}\left(\frac{P_{j k}^{D} h_{j k, j}^{2} d_{j k, j}^{-\alpha}}{P_{\max }^{C} h_{B, j}^{2} d_{B, j}^{-\alpha}+\sum_{\substack{j^{\prime}=1 \\
j^{\prime} \neq j}}^{N} \rho_{j^{\prime}} \sum_{\substack{k^{\prime}=1 \\
k^{\prime} \neq k}}^{L_{j}} \rho_{j^{\prime} k^{\prime}}^{\prime} P_{\max }^{D} h_{j^{\prime} k^{\prime}, j}^{2} d_{j^{\prime} k^{\prime}, j}^{-\alpha}+N_{0}}\right) \\
& R^{C} \simeq \log _{2}\left(\gamma^{\prime} C\left(P^{C}, P_{j k}^{D}\right)\right)= \\
& \log _{2}\left(\frac{P^{C} h_{B, C}^{2} d_{B, C}^{-\alpha}}{\rho_{j} \sum_{k=1}^{L_{j}} \rho_{j k} P_{j k}^{D} h_{j k, C}^{2} d_{j k, C}^{-\alpha}+\sum_{\substack{j^{\prime}=1 \\
j^{\prime} \neq j}}^{N} \rho_{j^{\prime}} \sum_{k=1}^{L_{j}} \rho_{j_{j k}} P_{\max }^{D} h_{j^{\prime} k, C}^{2} d_{j^{\prime} k, C}^{-\alpha}+N_{0}}\right)
\end{aligned}
$$

By using a log transformation of the power variables $P^{C} \rightarrow$ $2^{P^{C}}$ and $P_{j k}^{D} \rightarrow 2^{P_{j k}^{D}}$ [33], Eq. (22) is achieved.
By mathematical manipulation of (22), $f_{j}\left(P^{C}, P_{j k}^{D}\right)$ equals

$$
P^{C} \log _{2}\left(h_{B, C}^{2} d_{B, C}^{-\alpha}\right)-\log _{2}\left(\sum_{j} \zeta_{j} 2^{P_{j k}^{D}}+N_{0}\right),
$$

where $\zeta_{j}$ refers to the small scale fading coefficient of active interferer users for cellular links. Simply, the function $f_{j}\left(P_{j 1}^{D}, \ldots, P_{j L}^{D}\right)$ is in the form of a log-sum-exp function and hence is concave. Similarly, the function $g_{j k}\left(P_{j k}^{D}\right)$ is equal to

$$
\log _{2}\left(\frac{2^{P_{j k}^{D}} h_{j k, j}^{2} d_{j k, j}^{-\alpha}}{2^{P_{\max }^{C}} h_{B, j}^{2} d_{B, j}^{-\alpha}+\sum_{\substack{j^{\prime}=1 \\ j^{\prime} \neq j}}^{N} \rho_{j^{\prime}} \sum_{\substack{k^{\prime}=1 \\ k^{\prime} \neq k}}^{L_{j}} \rho_{j^{\prime} k^{\prime}}^{\prime} 2^{P_{\max }} h_{j^{\prime} k^{\prime}, j}^{2} d_{j^{\prime} k^{\prime}, j}^{-\alpha}+N_{0}}\right),
$$

where $g_{j k}$ is an affine and also a concave function. Thus, both the functions $f_{j}\left(P_{j 1}^{D}, \ldots, P_{j L}^{D}\right)$ and $g_{j k}\left(P_{j k}^{D}\right)$ fit in the definition of the pairwise separable convex program.

\section{REFERENCES}

[1] J. Liu, N. Kato, J. Ma, and J. Kadowaki, "Device-to-device communication in LTE-advanced networks: A survey," IEEE Commun. Surveys Tuts., vol. 17, no. 4, pp. 1923-1940, 4th quarter 2015.

[2] J. Liu, Y. Kawamoto, H. Nishiyama, N. Kato, and N. Kadowaki, "Device-to-device communications achieve efficient load balancing in lte-advanced networks," IEEE Wireless Communications, vol. 21, no. 2, pp. 57-65, 2014.

[3] J. Liu, S. Zhang, N. Kato, H. Ujikawa, and K. Suzuki, "Device-to-device communications for enhancing quality of experience in software defined multi-tier lte-a networks," IEEE Network, vol. 29, no. 4, pp. 46-52, 2015.

[4] J. Liu, H. Nishiyama, N. Kato, and J. Guo, "On the outage probability of device-to-device-communication-enabled multichannel cellular networks: An rss-threshold-based perspective," IEEE Journal on Selected Areas in Communications, vol. 34, no. 1, pp. 163-175, 2015.

[5] 3GPP TR 36.843 V12.0.1, "Study on LTE Device to Device Proximity Services; Radio Aspects ," Tech. Rep. V12.0.1. [Online]. Available: http://www.3gpp.org

[6] G. Fodor, E. Dahlman, G. Mildh, S. Parkvall, N. Reider, G. Mikls, and Z. Turanyi, "Design aspects of network assisted device-to-device communications," IEEE Commun. Mag., vol. 50, no. 3, pp. 170-177, Mar. 2012.

[7] S. Kazemi Rashed, R. Shahbazian, and S. A. Ghorashi, "Learningbased resource allocation in D2D communications with QoS and fairness considerations," Trans. Emerging Telecommun. Technol., vol. 29, no. 1, pp. 32-49, Jan. 2018

[8] J. Huang, Y. Yin, Y. Zhao, Q. Duan, W. Wang, and S. Yu, "A gametheoretic resource allocation approach for intercell device-to-device communications in cellular networks," IEEE transactions on emerging topics in computing, vol. 4, no. 4, pp. 475-486, 2014.

[9] S. Dominic and L. Jacob, "Distributed resource allocation for D2D communications underlaying cellular networks in time-varying environment," IEEE Commun. Lett., vol. 22, no. 2, pp. 388-391, Feb. 2018.

[10] M. Hasan and E. Hossain, "Distributed resource allocation in D2Denabled multi-tier cellular networks: An auction approach," in 2015 IEEE Conf. on Communications (ICC), 2015, pp. 2949-2954.

[11] A. Abedin and M. Rasti, "A distributed joint power control and mode selection scheme for D2D-enabled cellular systems," in 2016 IEEE Symp. on Computers and Communication (ISCC), 27-30 June 2016, pp. 1284-1289.

[12] H.-H. Nguyen, M. Hasegawa, and W.-J. Hwang, "Distributed resource allocation for D2D communications underlay cellular networks," IEEE Commun. Lett., vol. 20, no. 5, pp. 942-945, May 2016.

[13] F. Librino and G. Quer, "Distributed mode and power selection for non-orthogonal D2D communications: a stochastic approach," IEEE Transactions on Cognitive Communications and Networking, vol. 4, no. 2, pp. 232-243, June 2018.

14] N. Kumar, S. N. Swain, and C. S. R. Murthy, "A novel distributed Q-learning based resource reservation framework for facilitating D2D content access requests in LTE-A networks," IEEE Transactions on Network and Service Management, vol. 15, no. 2, pp. 718-731, June 2018. 


$$
f_{j}\left(P^{C}, P_{j k}^{D}\right)=\log _{2}\left(\frac{2^{P^{C}} h_{B, C}^{2} d_{B, C}^{-\alpha}}{\rho_{j} \sum_{k=1}^{L_{j}} \rho^{\prime}{ }_{j k} 2^{P_{j k}^{D}} h_{j k, C}^{2} d_{j k, C}^{-\alpha}+\sum_{\substack{j^{\prime}=1 \\ j^{\prime} \neq j}}^{N} \rho_{j^{\prime}} \sum_{k=1}^{L_{j}} \rho_{\prime_{j k}} 2^{P_{\max }^{D}} h_{j^{\prime} k, C}^{2} d_{j^{\prime} k, C}^{-\alpha}+N_{0}}\right) .
$$

[15] H. Zhao, K. Ding, N. I. Sarkar, J. Wei, and J. Xiong, "A simple distributed channel allocation algorithm for D2D communication pairs," IEEE Transactions on Vehicular Technology, vol. 67, no. 11, pp. 10960 $10969,2018$.

[16] A. Abrardo and M. Moretti, "Distributed power allocation for D2D communications underlaying/overlaying OFDMA cellular networks," IEEE Transactions on Wireless Communications, vol. 16, no. 3, pp. 1466-1479, 2017.

[17] C. Yang, J. Li, P. Semasinghe, E. Hossain, S. M. Perlaza, and Z. Han, "Distributed interference and energy-aware power control for ultra-dense D2D networks: A mean field game," IEEE Transactions on Wireless Communications, vol. 16, no. 2, pp. 1205-1217, 2017.

[18] S. Dominic and L. Jacob, "Fully distributed joint resource allocation in ultra-dense D2D networks: a utility-based learning approach," IET Communications, vol. 12, no. 19, pp. 2393-2400, 2018.

[19] R. Yin, C. Zhong, G. Yu, Z. Zhang, K. K. Wong, and X. Chen, "Joint spectrum and power allocation for D2D communications underlaying cellular networks," IEEE Transactions on Vehicular Technology, vol. 65, no. 4, pp. 2182-2195, 2016.

[20] S. Maghsudi and S. Stanczak, "Hybrid centralized-distributed resource allocation for Device-to-Device communication underlaying cellular networks." IEEE Trans. Vehicular Technology, vol. 65, no. 4, pp. 24812495, 2016.

[21] G. A. Safdar, M. Ur-Rehman, M. Muhammad, M. A. Imran, and R. Tafazolli, "Interference mitigation in D2D communication underlaying LTEA network," IEEE Access, vol. 4, pp. 7967-7987, Oct. 2016.

[22] X. Lin, J. Andrews, A. Ghosh, and R. Ratasuk, "An overview of 3GPP device-to-device proximity services," IEEE Commun. Mag., vol. 52, no. 4, pp. 40-48, Apr. 2014.

[23] J. Huang, C.-C. Xing, Y. Qian, and Z. J. Haas, "Resource allocation for multicell device-to-device communications underlaying 5g networks: A game-theoretic mechanism with incomplete information," IEEE Transactions on Vehicular Technology, vol. 67, no. 3, pp. 2557-2570, 2017.

[24] I. Sohn and S. H. Lee, "Distributed load balancing via message passing for heterogeneous cellular networks," IEEE Trans. Veh. Technol., vol. 65, no. 11 , pp. 9287-9298, Nov. 2016

[25] T. Lv, C. Wang, and H. Gao, "Factor graph aided multiple-symbol differential detection in the broadcasting phase of a network coding based UWB relay system," IEEE Trans. Veh. Technol., vol. 66, no. 6, pp. 5364-5371, June 2017.

[26] J. Dai, K. Niu, C. Dong, and J. Lin, "Improved message passing algorithms for sparse code multiple access," IEEE Trans. Veh. Technol., vol. 66, no. 11, pp. 9986-9999, Nov. 2017.

[27] F. Meyer, O. Hlinka, H. Wymeersch, E. Riegler, and F. Hlawatsch, "Distributed localization and tracking of mobile networks including non-cooperative objects," IEEE Trans. Signal and Inf. Process. over Networks, vol. 2, no. 1, pp. 57-71, Mar. 2016.

[28] A. Abrardo, M. Belleschi, P. Detti, and M. Moretti, "Message passing resource allocation for the uplink of multi-carrier multi-format systems," IEEE Trans. Wireless Commun., vol. 11, no. 1, pp. 130-141, Jan. 2012.

[29] M. Hasan and E. Hossain, "Distributed resource allocation for relayaided device-to-device communication: A message passing approach," IEEE Trans. Wireless Commun., vol. 13, no. 11, pp. 6326-6341, Nov. 2014.

[30] Y. Chen, J. Li, W. Chen, Z. Lin, and B. Vucetic, "Joint user association and resource allocation in the downlink of heterogeneous networks," IEEE Trans. Veh. Technol., vol. 65, no. 7, pp. 5701-5706, July 2016.

[31] M. Li, S. Zhang, N. Zhao, W. Zhang, and X. Wang, "Time-varying massive mimo channel estimation: Capturing, reconstruction, and restoration," IEEE Transactions on Communications, vol. 67, no. 11, pp. 75587572, 2019.

[32] A. Mohammed, S. Zhang, H. Li, N. Zhao, and X. Wang, "Capturing the sparsity and tracking the channels for massive mimo networks," IEEE Transactions on Vehicular Technology, vol. 69, no. 1, pp. 685-699.

[33] M. Chiang, "Balancing transport and physical layers in wireless mul- tihop networks: Jointly optimal congestion control and power control," IEEE J. Sel. Areas Commun., vol. 23, no. 1, pp. 104-116, Jan. 2005.

[34] S. Boyd and L. Vandenberghe, Convex optimization. Cambridge university press, New York, 2004.

[35] S. H. Lee and I. Sohn, "Message-passing strategy for joint user association and resource blanking in HetNets," IEEE Trans. Wireless Commun., vol. 17, no. 2, pp. 1026-1037, Feb. 2018.

[36] A. Behfarnia and A. Eslami, "Error correction coding meets cyberphysical systems: message-passing analysis of self-healing interdependent networks," IEEE Trans. Commun., vol. 65, no. 7, pp. 2753-2768, July 2017.

[37] F. R. Kschischang, B. J. Frey, and H.-A. Loeliger, "Factor graphs and the sum-product algorithm," IEEE Trans. Inf. Theory, vol. 47, no. 2, pp. 498-519, Feb. 2001.

[38] H. Wymeersch, Iterative receiver design. Cambridge University Press Cambridge, New York, 2007.

[39] C. C. Moallemi and B. Van Roy, "Convergence of min-sum messagepassing for convex optimization," IEEE Trans. Inf. Theory, vol. 56, no. 4, pp. 2041-2050, Apr. 2010.

[40] A. Gjendemsjø, D. Gesbert, G. E. Øien, and S. G. Kiani, "Binary power control for sum rate maximization over multiple interfering links," IEEE Trans. Wireless Commun., vol. 7, no. 8, pp. 3164-3173, Aug. 2008.

[41] F. Rothlauf, Design of modern heuristics: principles and application. Springer Science \& Business Media, Berlin, Heidelberg, 2011.

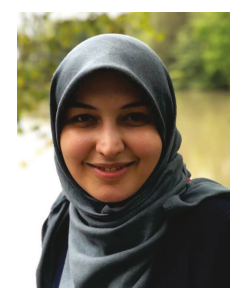

Salma Kazemi Rashed received her B.Sc. degree in Electrical Engineering, Communications from University of Tehran, in 2014 and her first M.Sc. degree in Telecommunications Engineering from ShahidBeheshti University, in 2017, Tehran, Iran. Salma also spent four years from $2014-2018$ as a research assistant at the Cognitive Radio Laboratory in Shahid-Beheshti University, Tehran, Iran, with the main research focus of interactive machine learning and distributed algorithms with applications in resource allocation of D2D networks. She received her second M.Sc. degree in Biomathematics, Bioinformatics and Computational Biology from Lund University, Lund, Sweden, in 2019. She is currently pursuing a Ph.D. in Department of Experimental Medical Science, AitsLab, in Lund University. Her main research interest is focusing on both the microscopy screening and the text-based genome-wide data analysis by applying machine learning and distributed optimization techniques.

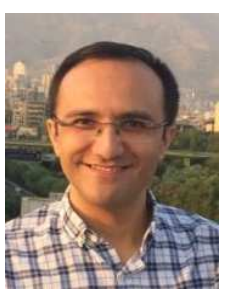

Reza Asvadi (M'11-SM'19) received the B.Sc. (with highest honors) and M.Sc. degrees in electrical engineering from $\mathrm{K}$. N. Toosi University of Technology and Sharif University of Technology, Tehran, Iran, in 2001 and 2003, respectively, and the Ph.D. degree from K. N. Toosi University of Technology in 2011. Since 2004 to 2006, he was a lecturer at Army Airforce University to fulfill his national service. He was a Postdoctoral researcher at the University of Oulu, Oulu, Finland, since 2012 to 2014. During the postdoc, he participated in many Academy of Finland and European Union (FP7) projects to research on iterative algorithms and information theoretical bounds over new emerging wireless networks. $\mathrm{He}$ is now an Assistant Professor in Shahid Beheshti University, Tehran, Iran. His research interests include coding and information theories and signal processing for wireless communications. He was a recipient of many Postdoctoral research grants including University of Alberta (2011-2012) and Carleton University (2014-2016) Postdoctoral Fellowships. 


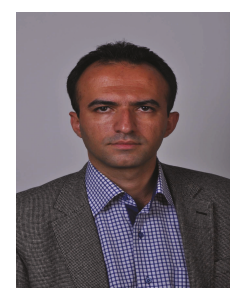

Siavash Rajabi received his B.Sc. and M.Sc. degrees in Electrical Eng. from the University of Tehran and K. N. Toosi University of Technology in 2003 and 2006, respectively. He obtained his Ph.D. degree from the Shahid Beheshti University, Tehran, Iran, in 2018. He joined the Hamedan University of Technology in 2018 where he is currently an Assistant Professor. His research interests include mathematical modeling and optimization of wireless communications focusing on $5 \mathrm{G}$ technology and Internet of Things applications.

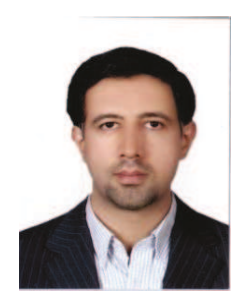

Seyed Ali Ghorashi received his BSc and MSc degrees in Electrical Engineering from the University of Tehran, Iran and his $\mathrm{PhD}$ degree from Kings College London, UK. He has worked for Samsung Electronics (UK) Ltd, Shahid Beheshti University, Middlesex University, Goldsmiths University of London and University of East London. $\mathrm{He}$ is a senior member of IEEE, holds US and international patents and has published over 120 technical papers mainly related to the applications of optimization, artificial intelligence and machine learning in positioning, internet of things and wireless communications.

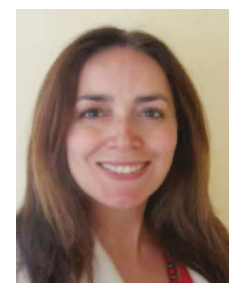

Maria Martini is Professor in the Faculty of Science, Engineering and Computing at Kingston University London, where she also leads the Wireless Multimedia Networking Research Group and is Course Director for the MSc in "Networking and Data Communications". She received the Laurea in electronic engineering (summa cum laude) from the University of Perugia (Italy) in 1998 and the Ph.D. in Electronics and Computer Science from the University of Bologna (Italy) in 2002. She has led the KU team in national and international research projects, funded by the European Commission (e.g., OPTIMIX, CONCERTO, QoENET, Qualinet), UK research councils (e.g., EPSRC, British Council, Royal Society), Innovate UK, and international industries. IEEE Senior Member (since 2007), Associate Editor for IEEE Signal Processing Magazine (20182021) and IEEE Transactions on Multimedia (2014-2018), she was lead guest editor for the IEEE JSAC special issue on "QoE-aware wireless multimedia systems" (2012), and editor for IEEE Journal of Biomedical and Health Informatics (2014), IEEE Multimedia (2018), Int. Journal on Telemedicine and Applications, among others. She is part of the NetWorld2020 ETP Expert Group, Board member of the Video Quality Expert Group (VQEG) and member of the IEEE Multimedia Communications technical committee, having served as vice-chair (2014-2016), chair (2012-2014) of the 3D Rendering, Processing, and Communications Interest Group, key member of the QoE and multimedia streaming IG. She currently chairs IEEE Standard WG P3333.1.4. Her current research interests include wireless multimedia networks, video quality assessment, event-based sensors, light field imaging, decision theory, machine learning and medical applications. She authored over 180 international scientific articles and book sections, international patents, and contributions to international standards (IEEE and ITU). 